\title{
L'étude de la polysémie verbale entre dérivation et invariance
}

\author{
Jacques François \\ Université de Caen, CRISCO EA 4255 \\ jacques.francois@unicaen.fr
}

Pour rendre compte de la polysémie verbale, Desclés \& alii (1998) distinguent « quatre attitudes méthodologiques »
i. strictement contextualiste (« effets de sens »),
ii. homonymique (« éclatement des significations »),
iii. dérivationnelle (« sens noyau et sens périphériques ») et
iv. schématisante (« à la recherche d'un invariant »).

A ces quatre démarches on peut adjoindre celle de la cartographie du sens.

On fera ici abstraction des deux premières attitudes peu représentées dans les travaux de la dernière décennie sur la polysémie verbale en français et on centrera l'exposé sur la comparaison entre les trois autres démarches dérivationnelle, schématisante et cartographique.

Dans la section 1, la démarche dérivationnelle sera illustrée par l'analyse contrastive de fr. ramper vs. ang. to crawl par Fillmore \& Atkins (2000) et l'analyse apparentée de fr. tenir vs. alld halten par François (2007).

Dans la section 2, on abordera trois variantes de la démarche schématisante, celle des schèmes sémanticocognitifs de J.P. Desclés, illustrée par son analyse de la polysémie du verbe arriver (2005), celle des schèmes conceptuels intégrés (héritiers de la démarche psychomécanique de J. Picoche) de M.L. Honeste, illustrée par ses deux études sur la polysémie du verbe venir, et celle des formes schématiques dans le cadre de la théorie des opérations prédicatives et énonciatives, illustrée par l'étude de la polysémie du verbe sentir par J.J. Franckel (2002, 2004).

La section 3 sera consacrée à la démarche cartographique et plus particulièrement au repérage des aires sémantaxiques, illustrée par deux études de la polysémie du verbe jouer, l'une (François, à paraître) consacrée au repérage manuel de l'aire de jouer une ouvre, l'autre (Jacquet, 2003) au repérage automatisé des emplois de jouer avec un régime prépositionnel (jouer à $\mathrm{N} /$ avec $\mathrm{N} / \boldsymbol{d e} \mathrm{N} / \operatorname{sur} \mathrm{N}$ ).

Enfin on comparera dans la section 4 quatre points de vue sur la polysémie du verbe monter défendus dans le cadre de la démarche schématisante, celui de J.P. Desclés (Desclés et al. 1998, Desclés \& Guentcheva 2005), celui de Lebas \& Cadiot (2003) dans le cadre de la théorie de « la construction extrinsèque du référent », celui de G. Jacquet (2004) sur la partie de l'espace sémantique de monter en combinaison avec différents compléments (un diamant, un escalier, un projet) et celui de P. Jalenques (2007) qui propose une interprétation de la commutation des cotextes de ce verbe apparentée à celle de M.L. Honeste $(2005 \mathrm{a}, \mathrm{b})$ pour pour la variété des cotextes de venir.

\section{La démarche dérivationnelle}

\subsection{Fillmore \& Atkins 2000 : fr. ramper vs. ang. to crawl}

On trouvera dans François (2007a:7-9) une présentation détaillée de l'article de Fillmore \& Atkins. J'insisterai ici sur les aspects de cette étude qui la distinguent le plus clairement de celle de François 
(2007b) abordée dans la section suivante. Les auteurs examinent en premier l'espace sémantique du verbe anglais to crawl et ils le décrivent selon une approche dérivationnelle à l'aide d'un réseau sémantique (Figure 5.5. de l'original) qui présente un noyau et des extensions. Ils passent ensuite à l'étude de l'espace sémantique du verbe français ramper dont certains des sens se recouvrent avec ceux de to crawl et ils le décrivent à l'aide d'un second réseau sémantique (Figure 5.8 de l'original) structuré également en noyau et extensions. Notre Figure 1 pointe sur les correspondances entre ces deux réseaux sémantiques.

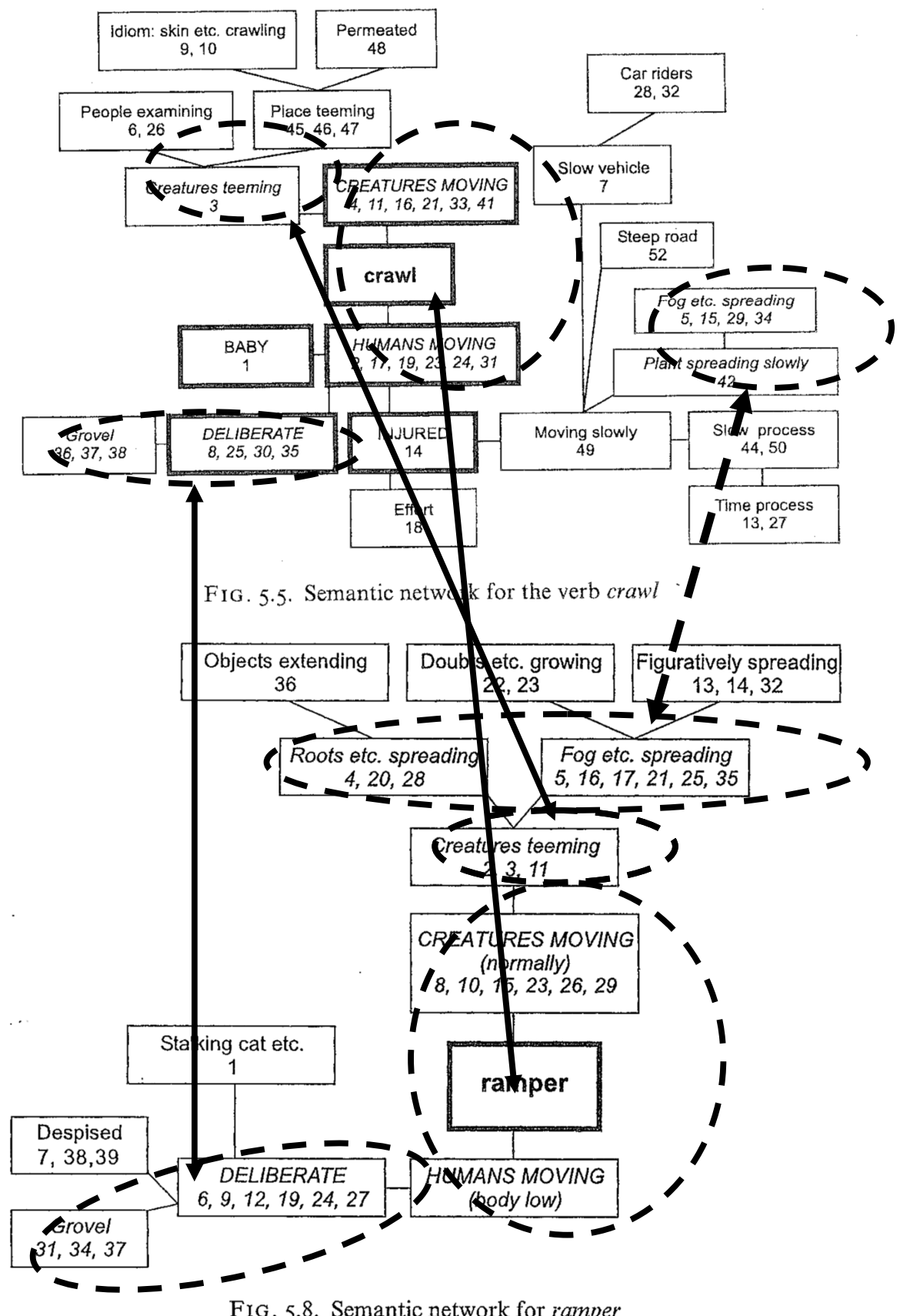

Figure 1 : Correspondances entre les réseaux sémantiques de to crawl et ramper selon Fillmore \& Atkins (2000) 
La comparaison entre les deux réseaux sémantiques de to crawl et de ramper révèle trois aires sémantiques communes (cases remplies en italiques dans les deux figures :

a) Un noyau sémantique commun constitué de deux variantes «creatures moving» et «humans moving » relatives à la sous-catégorisation du référent du sujet, lesquelles regroupent chacune plusieurs sous-types.

b) Parmi ces sous-types, «creatures teeming » (le grouillement de créatures) figure dans les deux réseaux pour la sous-catégorie [creature] et le sous-type de rang 1 DELIBERATE accompagné du sous-type de rang 2 «grovel» (être à plat ventre, ramper) pour la sous-catégorie [human] figure également dans les deux réseaux.

c) Enfin une correspondance moins claire entre les deux zones notées «Fogs etc. spreading » et deux zones caractérisées par une notation légèrement différente : «Plants spreading slowly » pour to crawl et « Roots etc. spreading » pour ramper. L'opacité de la correspondance a une double origine : d'une part les auteurs précisent p.104 que les racines rampent ne doit pas être traduit par roots crawl mais par roots creep, et d'autre part ils rattachent ces deux types d'emplois à la branche [HUMANS MOVING] $\rightarrow$ INJURED] $\rightarrow$ [Moving slowly] dans la figure 5.5. (to $\mathbf{c r a w l}$ ) et à la branche [CREATURES MOVING] $\rightarrow$ [creatures teeming] dans la figure 5.8 (ramper). On voit donc mal comment les représentations associées à the fog is / plants are crawling et le brouillard / des plantes rampe(nt) peuvent se superposer, compte tenu de leur disposition complètement différente dans l'un et l'autre réseau sémantique.

En tout état de cause, la conclusion est limpide : les deux réseaux représentés par les figures 5.5. et 5.8 ont une racine commune applicable à des créatures ou à des humains : "se déplacer par une activité musculaire tandis que le corps est tout proche du sol ou d'une autre surface», et des extensions partiellement analogues (en italiques) et partiellement divergentes (en caractères romains). La divergence principale tient, selon les auteurs, à l'évocation de deux images différentes : tandis que $\boldsymbol{t o}$ crawl « tend à véhiculer dans ses diverses manifestations figuratives le sens de membres qui par leur mouvement déplacent quelque chose qui s'apparente à un corps (...), ramper est centré sur le fait que l'entité rampante s'étend et couvre une surface de plus en plus grande $»^{1}$. Donc la synonymie partielle entre les deux verbes to crawl et ramper vient de ce que leurs deux espaces sémantiques présentent un noyau commun et des extensions divergentes. Cela distingue ce cas de celui de la synonymie partielle entre fr. tenir et alld halten, qui, d'après l'analyse contrastive de François (2007b), vient du recouvrement de certaines extensions de sens de l'un et l'autre verbes à partir de noyaux différents (cf. section 1.2). Ces deux configurations sont représentées dans la figure 2 (ci-après).

\subsection{François (2007b) : fr. tenir vs. alld halten}

Une autre sorte de démarche dérivationnelle est appliquée dans François (2007b) à l'analyse contrastive des verbes français tenir et allemand halten à partir de deux observations et de deux thèses, l'une sur le classement conceptuel des prédications verbales et l'autre sur la saillance relative des emplois contextuels :

a) La première observation est que, pour la majorité des bilingues français-allemand, la première traduction française de halten qui vient à l'esprit est tenir et vice-versa.

b) La seconde observation est que dans les dictionnaires monolingues de référence, l'un et l'autre verbe ont une longue liste d'emplois contextuels dont certains donnent lieu à la traduction par l'autre verbe.

c) La thèse sur le classement conceptuel des prédications verbales est que celui-ci se fonde sur le croisement de trois dimensions : notionnelle, aspectuelle et participative (ou actancielle). L'échelle aspectuelle pertinente pour le classement des emplois des deux verbes prévoit trois saisies :

(1) l'intransitionnalité (c'est-à-dire l'absence d'un changement attendu),

(2) la non-transitionnalité (ou stativité) et 
(3) la transitionnalité (éventuellement elle-même subdivisée en transitionnalité atélique (3a) et télique $(3 b))$.
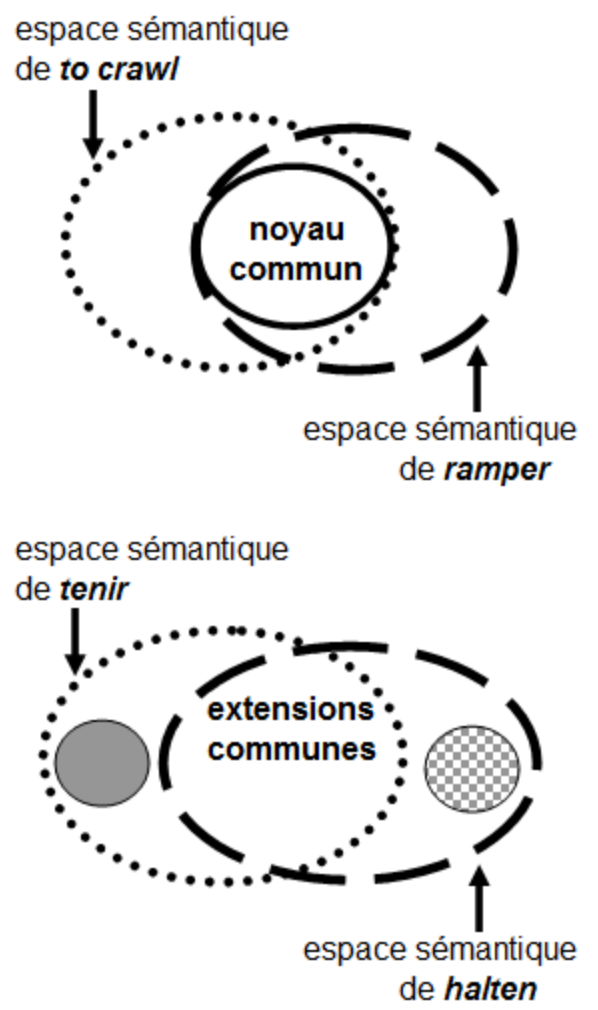

Figure 2: Les sources distinctes de la synonymie partielle entre angl. to crawl $\Leftrightarrow$ fr. ramper et fr. tenir $\Leftrightarrow$ alld halten

L'échelle de participation applicable aux deux verbes prévoit quatre saisies selon le degré d'investissement du référent du sujet grammatical en construction intransitive ou transitive indirecte ou de l'objet grammatical en construction transitive directe : (1) le référent est non-animé, (2) il est animé et non impliqué, (3) c'est un expérient faiblement impliqué, (4) c'est un agent (pouvent présenter par ailleurs le caracrère d'un expérient).

Tous les emplois répertoriés de chacun des deux verbes sont répartis dans une grille croisant les deux échelles (ce qui présente une certaine convergence avec la hiérarchie des schèmes sémantico-cognitifs statique $>$ cinématique $>$ dynamique de Desclés, cf. Section 2.1). On constate alors que 10 des 31 emplois répertoriés de tenir se rangent dans la classe \{non transitionnel / non agentif\} tandis que 14 des 42 emplois répertoriés de halten se situent dans la classe \{intransitionnel / agentif\}.

d) La thèse sur la saillance cognitive relative des emplois contextuels d'un verbe est que la classe combinatoire aspectuelle et participative qui regroupe la majorité des emplois est cognitivement la plus saillante et est assimilable au noyau sémantique de ce verbe.

Dans ce cadre méthodologique, la traduction fréquente de tenir par halten et vice-versa ne s'explique pas - contrairement à la traduction de crawl par ramper et vice-versa, cf. section 1.1 - par le recouvrement de leurs noyaux sémantiques, puisque ceux-ci diffèrent sur les deux échelles (\{non transitionnel / non agentif\} vs. \{intransitionnel / agentif\}), mais par deux dérivations inverses : une partie des emplois 'périphériques' de tenir correspond au noyau sémantique de halten et vice-versa. Le tableau 1 indique le nombre des emplois de tenir et halten dans chaque classe combinatoire. 


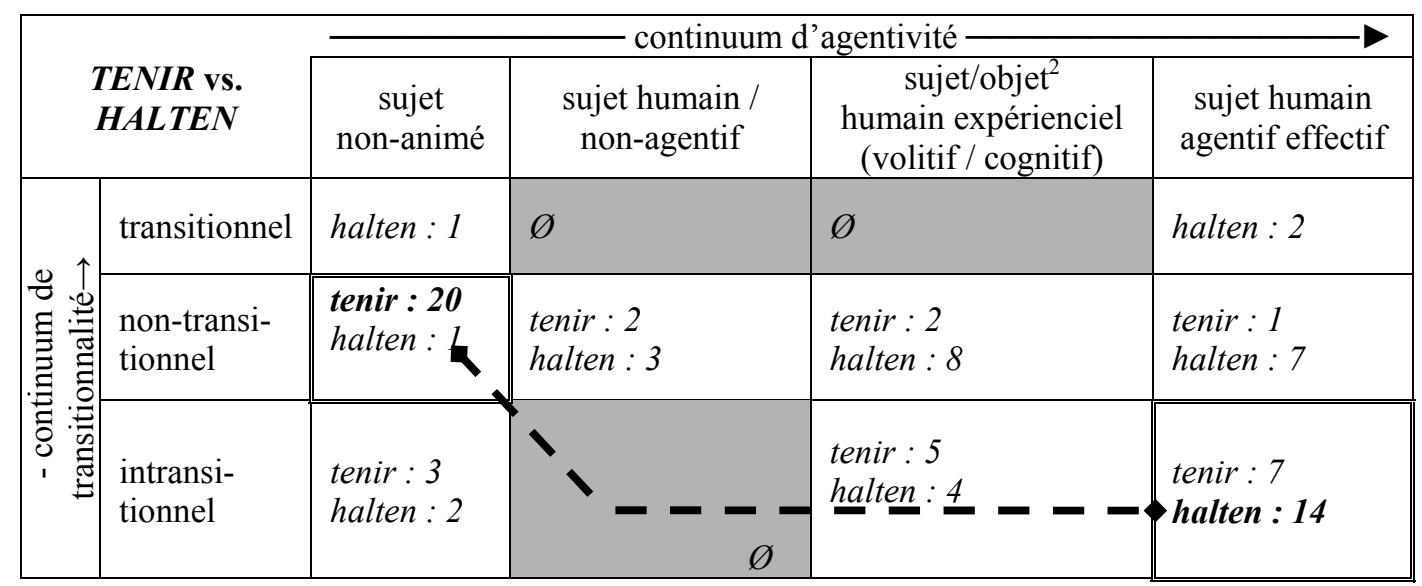

Tableau 1 : Localisation comparée du noyau sémantique des verbes tenir et halten sur les mêmes bases

Dans la classe \{non transitionnel / non agentif\} qui regroupe le plus de types d'emplois contextuels de tenir, on trouve un seul type d'emploi de halten, ex.

$$
\text { Le récipient tient l'eau } \Leftrightarrow \text { Das Faß hält das Wasser. }
$$

En revanche dans la classe \{intransitionnel / agentif\} regroupant le plus grand nombre de types d'emplois contextuels de halten, on rencontre 7 types d'emplois de tenir, ex.

Il se tient à une branche $\Leftrightarrow$ Er hält sich an einem Ast.

Tenir et halten sont représentés conjointement dans cinq autres classes (sans qu'ils soient partout traduisibles l'un par l'autre), et dans la classe \{intransitionnel / agentif\} qui accueille le noyau sémantique de halten, sur les sept emplois de tenir qui y figurent, cinq $(1,2,3,22,26)$ sont traduisibles par halten et un l'est par halten en combinaison avec le préfixe séparable auf-. D'autres combinaisons de halten avec les préfixes séparables durch- et fest- figurent également comme traductions des emplois 1, 22 et 26 de tenir, cf. Tableau 2.

\begin{tabular}{|l|l|l|}
\hline construction (illustration) & synonyme & traduction \\
\hline 1. Qqn tient qqn par la main & retenir & halten, festhalten \\
2. Qqn tient les yeux fermés & garder & halten \\
3. Qqn tient la preuve de la culpabilité de qqn & posséder & halten, haben, besitzen \\
6. Qqn tient un hôtel / un rôle & gérer, assumer & führen / leiten \\
22. Le bataillon a tenu trois jours & résister & halten / durchhalten \\
26. Qqn se tient à une branche & se cramponner & sich halten / festhalten \\
28. Qqn se tient des heures à la fenêtre & rester & sich aufhalten \\
\hline
\end{tabular}

Tableau 2: Les 7 emplois de tenir dans la classe \{intransitionnel / agentif\} et leurs traductions en allemand

Ce mode de raisonnement présente toutefois une faiblesse, celle d'admettre implicitement une corrélation entre la variété des emplois relevant d'une classe d'emplois particulière répertoriée par les dictionnaires monolingues et bilingues et la fréquence effective de ces emplois dans le discours quotidien. Pour conforter ce raisonnement, il faudrait pouvoir exploiter un corpus oral suffisamment étendu pour le français comme pour l'allemand. C'est une tâche qui reste à accomplir. 


\section{La démarche schématisante}

\subsection{L'approche des schèmes sémantico-cognitifs de J.P. Desclés, ex. toucher}

L'approche de Jean-Pierre Desclés et de ses collaborateurs ${ }^{3}$ se fonde sur le typage des entités participant au procès (entités individualisées, lieux, entités collectives ou passives, etc. cf. Desclés \& al. 1998 :34) et sur l'hypothèse de la validité cognitive d'une hiérarchie de trois types d'archétypes cognitifs: les archétypes d'état décrivant des situations telles que $\mathrm{X}$ est grand, $\mathrm{X}$ est supérieur à $\mathrm{Y}, \mathrm{X}$ ressemble à $\mathrm{Y}$, interviennent comme composantes d'archétypes cinématiques qui décrivent des transitions entre deux situations, telles que $\mathrm{X}$ augmente, $\mathrm{X}$ s'éloigne de $\mathrm{Y}, \mathrm{X}$ entre en contact avec $\mathrm{Y}$, et ceuX-ci à leur tour contribuent à la constitution d'archétypes dynamiques dans lesquels la transition entre deux états est mise en relation avec une instance causale (relation FAIRE) et éventuellement un agent (relation CONTR(ôle)), tels que $\mathrm{X}<$ agent $>$ fabrique $\mathrm{Y}, \mathrm{X}<$ cause $>$ produit $\mathrm{Y}, \mathrm{X}$ montre $\mathrm{Y}$ à $\mathrm{Z}$, etc.

L'analyse de la polysémie verbale implique «des formats représentationnels communs » (Desclés \& Guentcheva 2005 :95). Ce sont les schèmes sémantico-cognitifs (SSC) constitués à partir des trois types mentionnés ci-dessus d'archétypes cognitifs. La méthode consiste à «dégager des relations de spécification et d'abstraction entre schèmes représentatifs » (ibid.) avant de structurer par ces relations la famille des schèmes représentatifs impliquée dans la polysémie du verbe étudié. L'analyse peut - mais ne doit pas nécessairement - dégager un schème archétypal :

\footnotetext{
Dans un certain nombre de cas, les significations sont les spécialisations d'une signification beaucoup plus abstraite (représentée par un schème abstrait que nous appellerons archétype cognitif) qui sert d'élément organisateur au réseau des significations (Desclés et al. $1998: 29$ )

quand [un schème archétypal] existe, il représente la signification archétypale du verbe et de tous ses emplois (Desclés \& Guentcheva 2005 :95)
}

Je résumerai l'analyse proposée de la polysémie du verbe toucher par le diagramme ci-dessous. En premier lieu les auteurs (Desclés \& al. 1998) montrent comment l'archétype statique (que je noterai $\alpha$ ) intervient dans la construction de trois archétypes cinématiques (notés respectivement $\beta 1, \beta 2$ et $\beta 3$ ). Le premier met en œuvre un prédicat de mouvement entre deux localisations statiques, le second un prédicat de mouvement orienté et le troisième un prédicat de changement d'état. Ces trois archétypes cinématiques interviennent enfin comme constituants de différents archétypes dynamiques qui ont tous en commun la mise en œuvre d'un prédicat de causation FAIRE dans la portée d'un prédicat de contrôle agentif CONTR. Neuf types d'emplois sont distingués, l'emploi A est décrit à l'aide de l'archétype $(\alpha)$ de type statique. Les emplois $\mathrm{B}$ et $\mathrm{C}$ à l'aide respectivement des archétypes $\beta 1$ et $\beta 2$ de type cinématique. Les emplois $\mathrm{D}$ et $\mathrm{E}$ incorporent le schème $\mathrm{B}$ de mouvement dans un schème dynamique, l'emploi $\mathrm{F} y$ incorpore le schème $\mathrm{C}$ de mouvement orienté et les emplois $\mathrm{G}, \mathrm{H}$ et I l'archétype cinématique $(\beta 3)$ de changement d'état. Une opération d'abstraction intervient entre les schèmes $\mathrm{D}$ (contact physique) et $\mathrm{E}$ (contact verbal), tandis que les emplois $\mathrm{G}$ à I sont distingués par une opération de spécification des états initial et final en cause dans la transition (par exemple le passage pour le second actant y d'un état affectif «neutre» à un état affectif «non neutre »), cf. Figure 3. Au terme de l'analyse, la notion archétypale retenue est celle de contiguïté, contact ou frontière : «le contact est effectivement réalisé dans les situations statiques ou bien il tend à se réaliser dans les situations cinématiques et dynamiques » (Desclés \& al. 1998:41). 


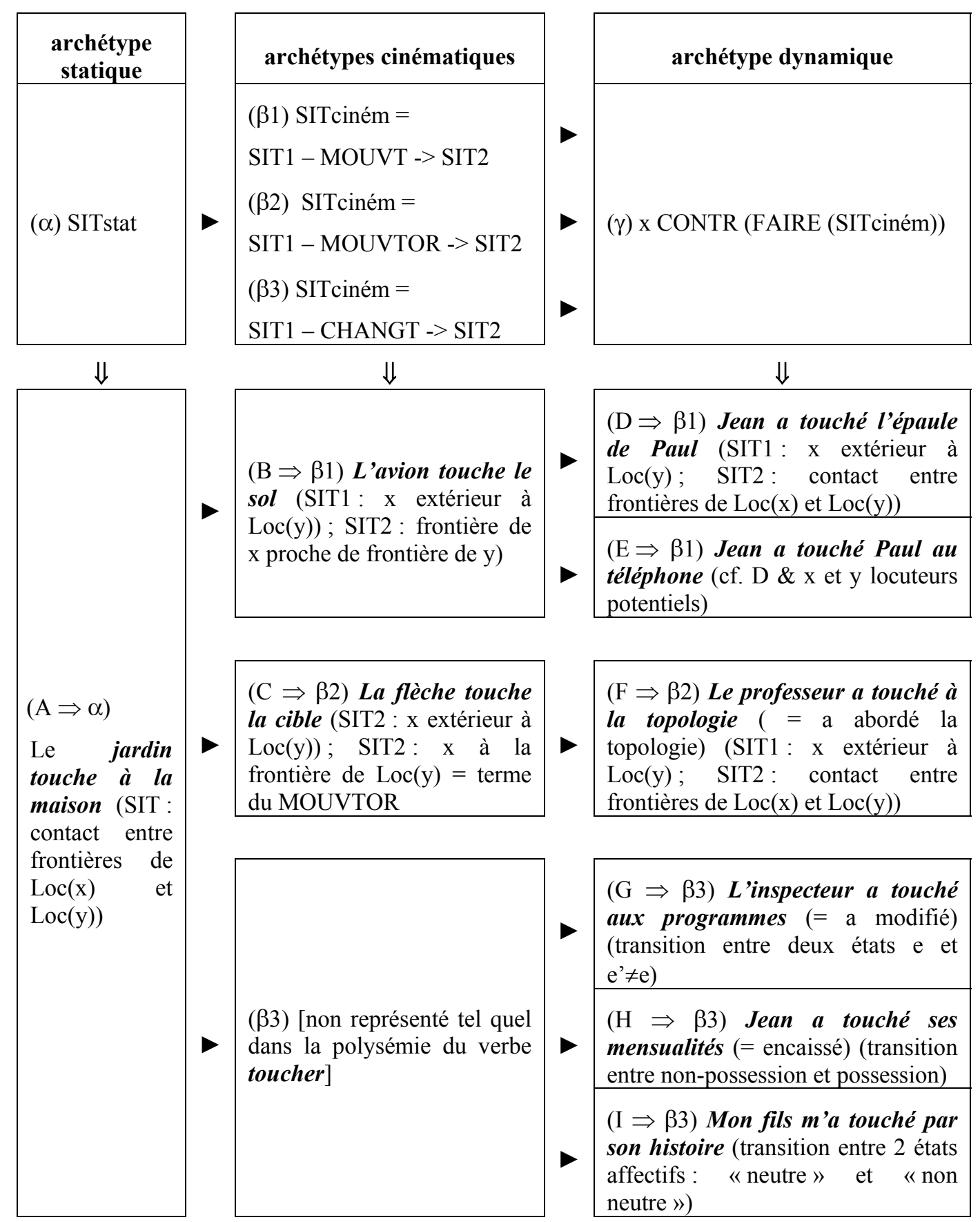

Figure 3 : Essai de représentation synoptique du réseau des schèmes sémantico-cognitifs associés à toucher selon Desclés et al. (1998:25-26)

Je formulerai trois remarques sur cette analyse :

i) En premier lieu, certaines descriptions sémantiques sont sujettes à débat, par exemple la différence entre L'avion touche le sol (Schème B) et La flèche touche la cible (Schème C) est assez obscure : l'avion ne se contente pas de frôler le sol, il entre pleinement en contact avec celui-ci tout comme la flèche par rapport à la cible, et il peut le faire violemment (ex. Quand au terme de sa chute l'avion a finalement touché le sol, il s'est brisé en deux et a explosé) tout comme la flèche. De même Jean a 
touché ses mensualités a pu impliquer le geste de prendre des billets de banque à l'époque où le règlement du salaire pouvait s'effectuer de la main à la main, mais à l'époque actuelle toucher un salaire signifie recevoir une ligne de crédit sur un compte bancaire et n'implique donc aucun FAIRE ni aucun CONTRÔLE de la part du prime actant. Je disposerais donc plutôt le schème $\mathrm{H}$ dans la colonne cinématique

ii) Cette analyse ne permet pas de dégager les conditions de sélection entre les deux constructions $\mathrm{N}: \mathrm{x}$ touche $\mathrm{N}$ :y (schème statique $\mathrm{A}$, schèmes dynamiques $\mathrm{F}$ et $\mathrm{G}$ ) et $\mathrm{Nx}$ touche à $\mathrm{N}$ :y (schèmes cinématiques $\mathrm{B}$ et $\mathrm{C}$, schèmes dynamiques $\mathrm{D}, \mathrm{E}, \mathrm{H}$ et $\mathrm{I}$ ). En particulier, il est curieux que le schème cinématique $\mathrm{C}$ $(\mathrm{N}: \mathrm{x}$ touche $\mathrm{N}: \mathrm{y})$ entre dans la construction du schème dynamique $\mathrm{F}(\mathrm{N}: \mathrm{x}$ touche à $\mathrm{N}: \mathrm{y})$. On s'attendrait à ce que la préposition $a ̀$ soit présente ou absente dans les deux constructions liées par cette relation d'emboitement.

iii) J.P. Desclés s'est explicitement distancié des analyses de R. Jackendoff $(1983,1990,2002)$ en expliquant que son usage des relations topologiques n'implique aucun privilège accordé aux relations spatiales dans les archétypes cognitifs. Cependant dans l'architecture des primitifs cognitifs les deux démarches sont étroitement apparentées. L'impression générale est que si une représentation spatiale est en cause, elle fonctionne généralement comme réalisation primaire de l'archétype cognitif à partir de laquelle d'autres réalisations sont dérivées par l'opération d'abstraction ${ }^{4}$. En revanche si aucune représentation spatiale n'est en cause, par exemple en cas de changement d'état, c'est l'opération de spécification du changement qui distingue les différentes réalisations schématiques (ici entre les schème G, H et I). De ce fait, la démarche de J.P. Desclés paraît globalement plus proche de celle de Jackendoff que de celle des défenseurs des «formes schématiques », par exemple J.J. Franckel \& Lebaud (1990). L'analyse du verbe toucher proposée en 2004 par D. Lebaud, qui conclut que "Toucher marque l'articulation de la localisation d'un terme $\mathrm{X}$ par un terme $\mathrm{Y}$ à l'autonomie première de $\mathrm{X}$ et $\mathrm{Y}$ » (p.71), plus détaillée et d'un accès plus délicat que celle de Desclés, se caractérise par un double souci d'une part de suivre de plus près la variation syntaxique et d'autre part de dériver les différentes réalisations syntactico-sémantiques directement de la forme schématique et non les unes des autres.

\subsection{L'approche des schèmes conceptuels intégrés et l'héritage psychomécanique chez M.L. Honeste, ex. venir (2005a, 2005b)}

M. L. Honeste, dont la conception de la polysémie verbale s'inspire initialement des analyses psychomécaniques de J. Picoche (1986), a consacré deux études parues la même année (2005a,b) au verbe venir. Je suivrai l'argumentation qu'elle développe dans sa contribution au colloque Les périphrases verbales (2005a). L'auteure commence (p.293) par critiquer la théorie de l'extension métaphorique en faisant valoir que pour rendre compte de l'expression les jours qui viennent il faudrait soit spatialiser l'interprétation du SN les jours, soit déspatialiser celle du verbe venir. Elle présente la polysémie comme une illusion issue du rattachement "au signifié de l'item des éléments de sens véhiculés par d'autres items de l'énoncé » (p.294). Pour mettre à nu le signifié "propre » et invariant du verbe venir, il faut donc faire abstraction de tout ancrage référentiel.

La méthode proposée par M.L. Honeste consiste à repérer dans chaque cotexte la part du sens construit qui est imputable à ce cotexte. Pour cela il est utile de faire varier les cotextes pour un même verbe et les verbes pour un même cotexte. C'est ainsi que l'énoncé Max VIENT DE Paris donne matière à comparaison d'une part avec Max VIENT A Paris et d'autre part avec Max EST DE Paris. L'auteure examine successivement les emplois spatiaux qui résultent de la présentation du verbe dans un cotexte d'arguments spatiaux, puis les emplois temporels, comparant $\mathrm{X}$ vient de $\mathrm{A}(\mathrm{SN})$ et $\mathrm{X}$ vient de vbINF (ex. Max vient de Paris vs. Max vient de partir), $\mathrm{X}$ vient à $\mathrm{B}(\mathrm{SN})$ et $\mathrm{X}$ vient à $\mathrm{B}(\mathrm{N}, \mathrm{b} I \mathrm{NF})$ (ex. Max vient à Paris vs. Max vient à penser à Marie / Marie lui vient (à l'esprit)), ainsi que $\mathrm{X}$ vient $\mathrm{C}$ (vbINF), ex. Max vient travailler. Enfin elle aborde les emplois notionnels, ceux où $\mathrm{X}$ ne désigne plus un animé, ex. Ma cicatrice vient d'une blessure, Tout notre mal vient de ne pouvoir être seuls et ceux qui impliquent une « situation préalablement définie dans le contexte» (p.305) sous la forme du clitique en, ex. Max en vient aux mains / à penser que $\mathrm{P}$. A chaque stade de l'analyse, elle dépouille le signifié de venir d'un 
nouveau trait, par exemple de la valeur de «passé récent» (Max vient de partir), de rapprochement du lieu et du moment de l'énonciation (Max vient travailler) ou de la relation de cause à effet (Ma cicatrice vient d'une blessure), toutes valeurs véhiculées par l'un ou l'autre élément du cotexte (préposition $\grave{a}$ vs. $\boldsymbol{d e}$, sous-catégorisation animé vs. non animée du sujet, catégorisation de l'objet prépositionnel : SN vs. vbINF).

M.L. Honeste propose finalement le schéma conceptuel suivant qui se limite à la représentation de la tension vers un centre déictique . Le schéma conceptuel de venir (p.306), se présente ainsi :

\section{$\checkmark \mathrm{X}$ tend vers le centre déictique (point de référence implicite) :}

- le procès venir n'intègre ni la situation initiale $\mathrm{A}$ de $\mathrm{X}$ avant le procès, ni la situation finale $\mathrm{B}$ après le procès ;

- l'énoncé peut rendre prégnant $\mathrm{A}$ au moyen d'un complément introduit par de ou $\mathrm{B}$, au moyen d'un complément introduit par à, vers, chez ou autre ;

- A est nécessairement distinct du centre déictique ; il est en lien étroit avec le procès venir ;

- B se situe nécessairement au centre déictique ; il est coupé du procès venir ;

- un procès $\mathrm{C}$ peut constituer un prolongement du procès venir; il a lieu soit au centre déictique, soit ultérieurement ;

- la nature et les relations de $\mathrm{X}, \mathrm{A}, \mathrm{B}$ et $\mathrm{C}$ déterminent par inférence le domaine d'application contextuel de l'énoncé.

Moule syntaxique : [X vient (de A) (à B) (C)]

La méthode préconisée par M.L. Honeste peut donc être dite distributionnelle et compositionnelle : distributionnelle, puisqu'elle vise à relier chaque acception à des propriétés du cotexte, et compositionnelle, puisqu'elle suppose que la sélection de la préposiion à vs. de, la sous-catégorisation du sujet et la catégorisation nominale ou infinitivale de l'objet prépositionnel sont en elles-mêmes porteuses d'un sémantisme propre à 'informer' l'invariant sémantique du verbe venir (signifié de puissance dans la terminologie guillaumienne traditionnelle, schéma conceptuel dans l'article recensé). Cette méthode est étroitement apparentée à celle qu'emploie également $\mathrm{P}$. Jalenques (à paraître 1,2, cf. §4.5). Elle est convaincante, mais on regrette seulement que l'auteure ne discute pas l'analyse en termes de forme schématique proposée antérieurement par Franckel \& Lebaud (1990:161-177), alors même que, par l'abandon de la notion de subduction proposée par J. Picoche (1986) « au profit d'une hypothèse radicale de l'unité du signe » (2005b: 81), elle fait un pas important en direction de la théorie des formes schématiques et s'éloigne simultanément de la conception de J.P. Desclés privilégiant des opérations d'abstraction.

\subsection{L'approche des formes schématiques, ex. sentir (Franckel 2002, 2004) ${ }^{5}$}

Dans son analyse de la polysémie du verbe sentir en termes de forme schématique, Franckel (2004, développant 2002) retient comme invariant l'existence d'un déclencheur $\mathbf{d}$ extérieur à $\mathbf{S}$, le sujet du sentir, mais dont le contenu qualitatif vient de $\mathbf{S}$ sans que celui-ci maîtrise ni le déclenchement de la représentation ni la représentation elle-même (p.108-9). La forme schématique a la forme

Sentir prédique l'existence pour un sujet (S) de quelque chose (e) qu'il spécifie comme le déclencheur (d) d'une représentation (P) dont il devient le site

Les différents emplois de sentir sont dérivés - comme pour ceux de passer, cf. §I-1.3 - en deux temps, d'abord par le jeu des variations liées aux différents modes de mise en œuvre de la forme schématique (par exemple dans Cette table sent la cire, l'existence d'un processus de déclenchement « dans lequel le déclencheur n'est ni identifié, ni identifiable », p.117) et ensuite par la corrélation entre les paramètres de 
la forme schématique et le schéma de lexis ${ }^{6}$, par exemple la subtile différence entre Ça sent! (degré 'zéro' de la détermination qualitative de $\mathrm{P}$, la représentation déclenchée) et Ça sent quelque chose (degré 'minimal' de cette détermination).

L'article débouche sur un intéressant parallèle entre l'éventail des emplois de sentir et celui des emplois du nominal sens, supposé mettre en jeu les mêmes paramètres ( $\mathrm{S}, \mathrm{d}$ et $\mathrm{P})$.

\section{La démarche cartographique et le repérage des aires sémantaxiques : l'exemple du verbe jouer}

La démarche cartographique a été impulsée à l'origine par B. Victorri (cf. Victorri \& Fuchs 1996). Elle a d'abord donné lieu à des travaux de sémantique computationnelle portant sur le lexique nominal, adjectival et verbal dont la contribution de Jacquet, Venant \& Victorri au volume Sémantique et traitement automatique des langues (Enjalbert, dir. 2005) donne un résumé synthétique. Dans un second temps, elle a fourni la matière de mon étude Pour une cartographie de la polysémie verbale (2007a) qui examine dans une perspective lexicographique la polysémie de onze verbes français (affecter, appeler, changer, charger, couper, manquer, monter, rapporter, relever, reposer et voler) ainsi que d'articles consacrés également à la polysémie des verbes affecter (François 2008), compter (François 2009) et jouer (François 2007c, à par.).

Je vais illustrer les deux approches, lexicographique et computationnelle, en comparant deux analyses syntactico-sémantiques d'une partie de la polysémie du verbe jouer, d'abord celle, lexicographique, de François (à par.), puis celle, computationnelle, de Jacquet (2004).

\subsection{Jouer une œuvre (François 2007c, à par.)}

Le propos de ces deux études est d'exploiter le Dictionnaire Électronique des Synonymes (DES) du CRISCO pour rendre compte de différents emplois du verbe jouer en rapport avec la musique et l'art lyrique, à savoir jouer un morceau, jouer un opéra, jouer d'un instrument et jouer un rôle. La question est celle du repérage de la partie de l'espace sémantique du verbe jouer visualisée par le DES en rapport avec chacun de ces emplois. Il faut d'abord rappeler que l'espace sémantique du verbe jouer est organisé à partir des interdistances entre les 90 cliques de ses 88 synonymes et que cet espace a autant de dimensions qu'il y a de synonymes, donc 88 dimensions. Chaque clique étant une combinaison de l'item examiné et de synonymes entretenant tous une relation synonymique mutuelle, elle constitue une coordonnée de sens dans l'espace multidimensionnel de l'item en cause.

La méthode préconisée consiste à tirer parti de la possibilité de visualiser l'espace commun à plusieurs items. On part de l'observation que le meilleur synonyme de X joue un morceau est X INTERPRETE un morceau. Pour X (ex. une troupe lyrique) joue un opéra c'est X REPRESENTE un opéra, pour X joue d'un instrument c'est X PRATIQUE / MANIE un instrument et pour X joue un rôle (dans une ouvre lyrique, théâtrale ou cinématographique), c'est X interprète un rôle. La figure 3 (page suivante) représente (sur le plan de projection 1*2 proposé par défaut par le logiciel VisuSyn) la visualisation de l'espace composé des verbes jouer, jouer de ${ }^{7}$, manier, pratiquer, interpréter et représenter.

On constate premièrement que - comme souhaité - jouer occuper une position centrale dans cet espace composé. Deuxièmement manier et pratiquer partagent avec jouer un espace commun comportant les cliques \{jouer, manier, pratiquer, tenir\} et \{jouer, manier, pratiquer, user\}. En revanche les cliques \{agiter, jouer, manier\} et \{jouer, manier, toucher, tripoter\} excluent pratiquer et les cliques \{jouer, pratiquer, s'entraîner $\}$ et \{exécuter, faire, jouer, pratiquer, tenir $\}$ excluent manier. Troisièmement, il en est de même pour interpréter et représenter qui ont en commun avec jouer un sous-espace caractérisé par les cliques \{faire, interpréter, jouer, représenter\} et \{incarner, interpréter, jouer, représenter\}. Comme précédemment les cliques \{exécuter, faire, interpréter, jouer $\}$, \{interpréter, jouer, tourner $\}$ et \{créer, faire, interpréter, jouer\} excluent représenter, tandis que les cliques \{agir, faire, jouer, représenter\} et \{donner, exposer, jouer, représenter\} excluent de leur côté le verbe interpréter. 
Sur cette base on peut calculer pour chaque clique son taux d'accord avec une construction donnée en testant la compatibilité de chacun des synonymes qui la constituent avec cette construction. Ainsi pour $X$ joue un morceau la clique 253 \{jouer, pianoter $\}$ présente un seul synonyme, lequel est substituable à jouer dans la construction (X pianote un morceau). La clique 253 présente donc un taux d'accord de $100 \%$. La clique 98 \{faire, créer, faire, interpréter, jouer\} présente trois synonymes dont deux sont substituables à jouer dans la construction $\left(X\right.$ crée $^{8} /$ interprète / fait un morceau). Le taux d'accord est de 2/3 (67\%). Il en est de même pour la clique 80 \{exécuter, faire, interpréter, jouer\}. 


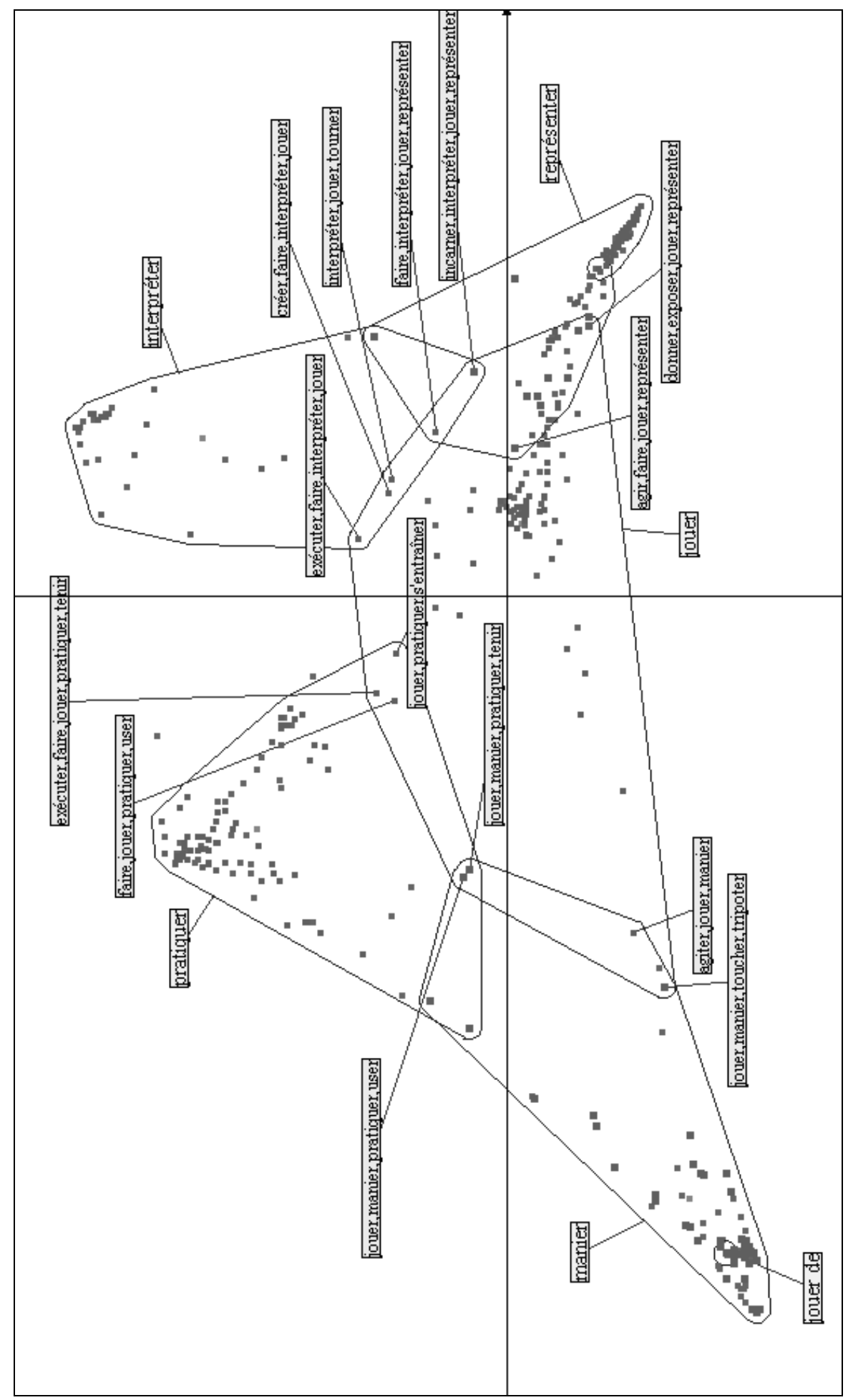

Figure 3: Visualisation sur le plan 1x2 par défaut de l'espace composé de \{jouer (de), interpréter, manier, pratiquer et représenter\} 
La figure 4 représente par zoom l'espace commun aux verbes jouer et interpréter et la position des trois cliques.

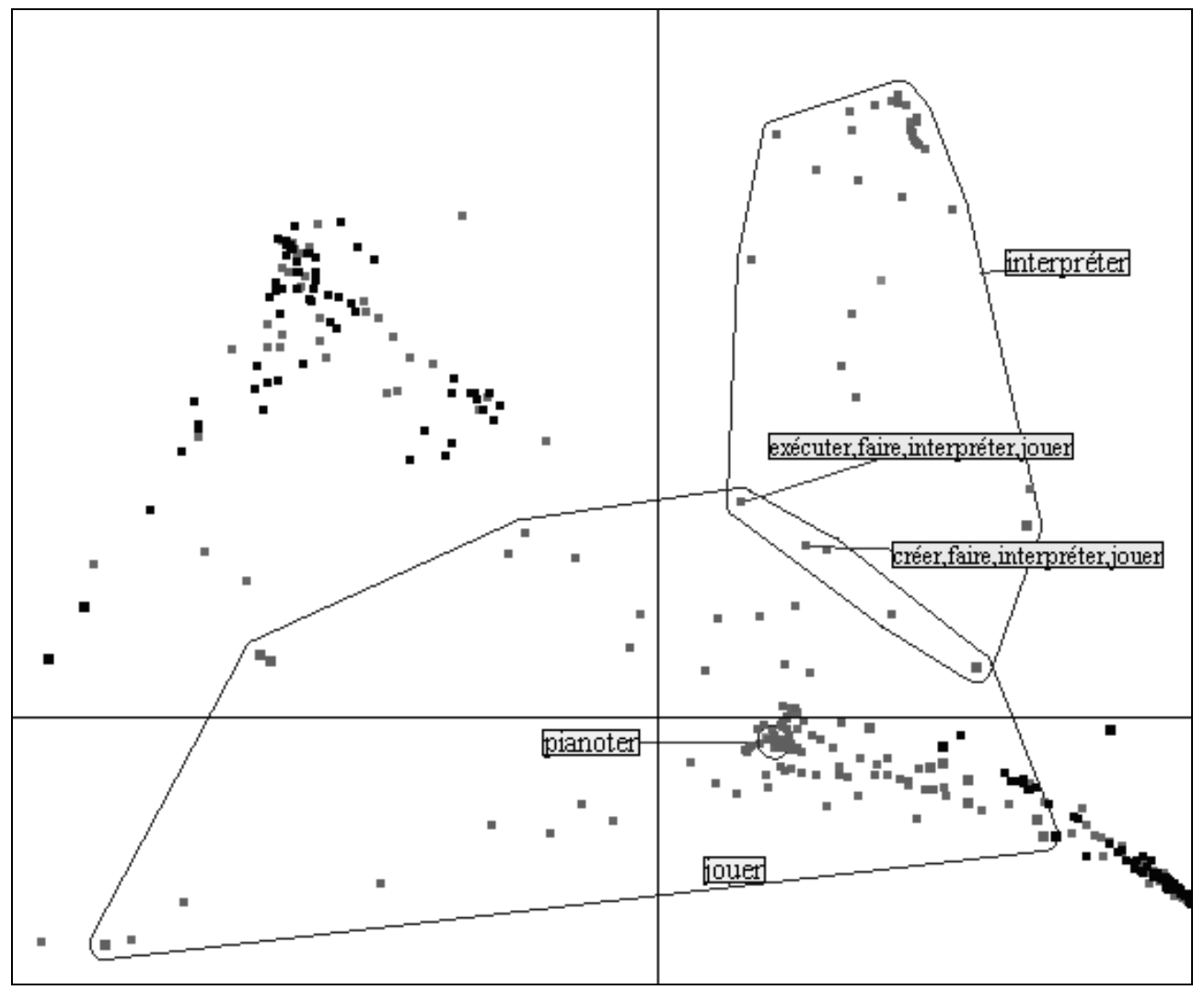

Figure 4 : Visualisation par zoom sur le plan 1x2 de l'aire sémantaxique de qn joue un morceau / opus

La clique 253, qui ne comporte pas interpréter, se situe plus près du centre de l'espace du verbe jouer. La même méthode est appliquée dans François (à paraître) aux trois autres constructions $X$ joue un opéra / d'un instrument / un rôle. On constate entre autres que la clique 232 \{incarner, interpréter, jouer, représenter $\}$ est majoritairement compatible à la fois avec les constructions jouer un opéra et jouer un rôle, et cela pour des raisons différentes. En effet incarner est le seul verbe exclu de $X \sim$ un opéra et représenter le seul verbe exclu de $X \sim$ un rôle. Ces deux cliques se recouvrent donc partiellement, avec une extrémité orientée vers la représentation d'une œuvre lyrique (le sujet réfère à un orchestre, un chef d'orchestre, un metteur en scène ou une institution lyrique) et une autre extrémité orientée vers l'incarnation d'un rôle (le sujet réfère à un interprète) :

\begin{tabular}{|l|l|l|l|l|}
\hline $\begin{array}{l}\text { INCARNATION } \\
\text { (X joue un rôle })\end{array}$ & incarner & interpréter & représenter & \\
\hline & incarner & interpréter & représenter & $\begin{array}{l}\text { REPRÉSENTATION } \\
\text { (X joue un opéra })\end{array}$ \\
\hline
\end{tabular}

En conclusion, il s'avère donc possible d'attribuer à chaque clique de synonymes du verbe jouer un ou plusieurs cadre(s) prédicatif(s) dominant(s), celui ou ceux que partage la totalité ou la majorité ${ }^{9}$ des verbes constituant la clique. 


\subsection{Jouer et les régimes prépositionnels (Jacquet 2003)}

Guillaume Jacquet - dont j'évoquerai plus loin la thèse (2004) à propos de la polysémie du verbe monter - a consacré un article (2003) au repérage automatique du sous-espace sémantique du verbe jouer correspondant aux différents régimes prépositionnels $\mathrm{X}$ joue sur $\mathrm{Y}, \mathrm{X}$ joue avec $\mathrm{Y}$ et $\mathrm{X}$ joue à/de $\mathrm{Y}$. Il commence par visualiser l'espace sémantique de jouer qui sur le plan $1 * 2$ par défaut présente trois extrémités, supérieure avec la clique \{jouer, miser, ponter, risquer\} qui suggère la pratique des jeux d'argent, inférieure gauche avec la clique \{batifoler, folâtrer, jouer, plaisanter, s'amuser\} suggérant le jeu comme détente et inférieure droite avec la clique \{copier, imiter, jouer, mimer, reproduire, simuler\} qui évoque le jeu mimétique (Figure 4.6. de Jacquet 2004 ci-dessous).

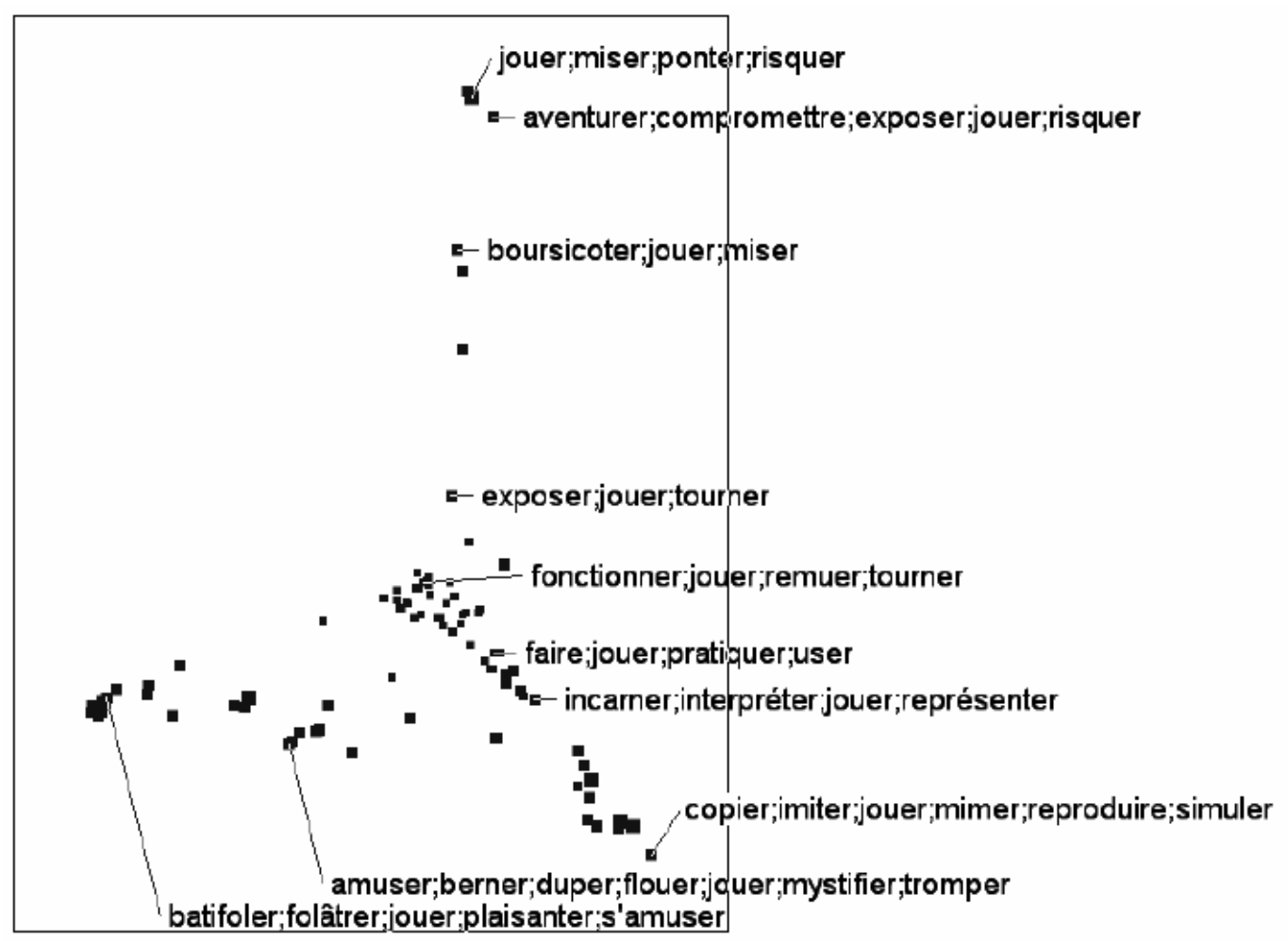

Figure 4.6 : Espace sémantique du verbe jouer

Figure 5 extraite de Jacquet (2003)

Ensuite il repère dans un corpus journalistique catégorisé tous les cotextes droits de l'item jouer du type sur SN, il en extrait la tête nominale, recherche les synonymes de jouer qui cooccurrent avec cette tête nominale à droite (avec ou sans préposition) et calcule le degré de compatibilité de chaque clique de synonymes avec ce type de cotextes, ce qui lui permet de visualiser ${ }^{10}$ le sous-espace sémantique de jouer sur (Figure 2 de Jacquet 2003). Les verbes en gras sont ceux qui cooccurrent avec les mêmes têtes nominales que jouer sur SN.

Ce sous-espace apparaît constitué d'une aire primaire regroupant quatre cliques (dans la zone inférieure) et d'une aire secondaire dissociée qui en regroupe deux (dans la zone supérieure). Seule la clique \{boursicoter, jouer, spéculer, tripoter\} présente une compatibilité élevée avec le cotexte sur N sans appartenir à aucune de ces deux aires. Elle occupe géométriquement une position intermédiaire, mais sémantiquement elle se rattache plutôt à l'aire secondaire, car la sous-catégorisation de la tête nominale 
introduite par sur est ici clairement l'objet d'un pari ou d'une spéculation. En revanche l'unité sémantique de l'aire primaire parait douteuse.

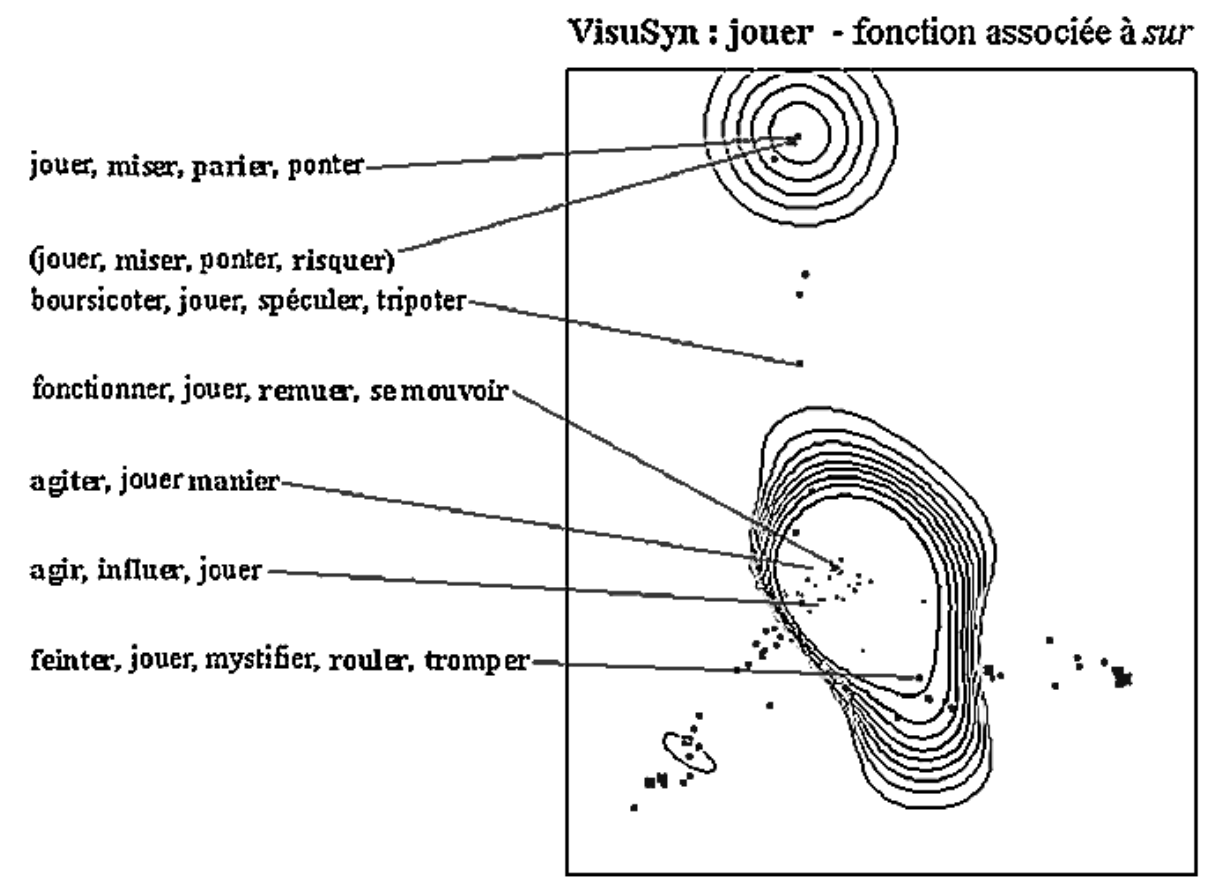

Figure 2 : sous-espace sémantique de « jouer sur»

Figure 6 extraite de Jacquet (2003)

La même méthode est appliquée ensuite au cotexte avec SN. Seules quatre cliques ${ }^{11}$ se révèlent globalement compatibles avec le cotexte avec $\mathrm{N}$. Trois d'entre elles constituent une aire primaire fortement compatible, tandis que la quatrième : \{aventurer, compromettre, exposer, hasarder, jouer, risquer\} constitue une aire secondaire à elle seule. Il est à noter que chacune de ces cliques est plus particulièrement compatible avec une sous-catégorie sémantique particulière de avec SN. Ainsi la clique ci-dessus est compatible avec le cadre prédicatif $\mathrm{SN}<\mathrm{x} 1$ :hum $>$ joue avec $\mathrm{SN}<\mathrm{x} 2$ :argent $>$ et la clique \{jouer, manier, toucher, tripoter\} avec le cadre $\mathrm{SN}<\mathrm{x} 1$ :hum $>$ joue avec $\mathrm{SN}<\mathrm{x} 2$ :instrument $>$ (cf. Figure 7 ci-après).

Jacquet compare finalement les cliques qui se révèlent globalement compatibles avec comme cotexte droit soit $\boldsymbol{d e}(\boldsymbol{l a}) / \boldsymbol{d u} / \boldsymbol{d e s} \mathrm{N}$ soit à $(\boldsymbol{l a}) / \boldsymbol{a u}(\boldsymbol{x}) \mathrm{N}$. Les premières (Figure 8 , originellement Figure 4 de Jacquet 2003) constituent deux aires reliées l'une à l'autre. L'aire primaire regroupe cinq cliques tandis que l'aire secondaire se limite à la clique \{abuser, berner, duper, jouer, mystifier, rouler . Les secondes (Figure 9, originelemment Figure 5 de Jacquet 2003) constituent une aire primaire avec cinq cliques qui tournent autour de deux idées distinctes, l'interprétation (jouer (qch) à un instrument) et le divertissement (jouer $\grave{a}$ un jeu $)$ et une aire secondaire avec trois cliques en rapport avec les jeux d'argent comme précédemment pour jouer sur SN.

En conclusion, on constate que, si l'entreprise de Jacquet est stimulante, elle se heurte cependant à des obstacles en raison de l'insuffisante caractérisation du cotexte droit par les têtes nominales. En effet, en particulier pour jouer de (la)/du/des N, le morphème introducteur peut être soit une préposition contractée (ex. jouer des coudes) soit un déterminant partitif (ex. jouer des sommes d'argent importantes), ce qui caractérise des cadres prédicatifs complètement différents. Cependant cette méthode de repérage de sousespaces sémantiques - apparentée dans son principe à celle que j'ai appliquée manuellement, cf. section 3.1 - se révèle éclairante pour les cadres prédicatifs transitifs du verbe monter, comme nous le verrons en section 4.3 . 


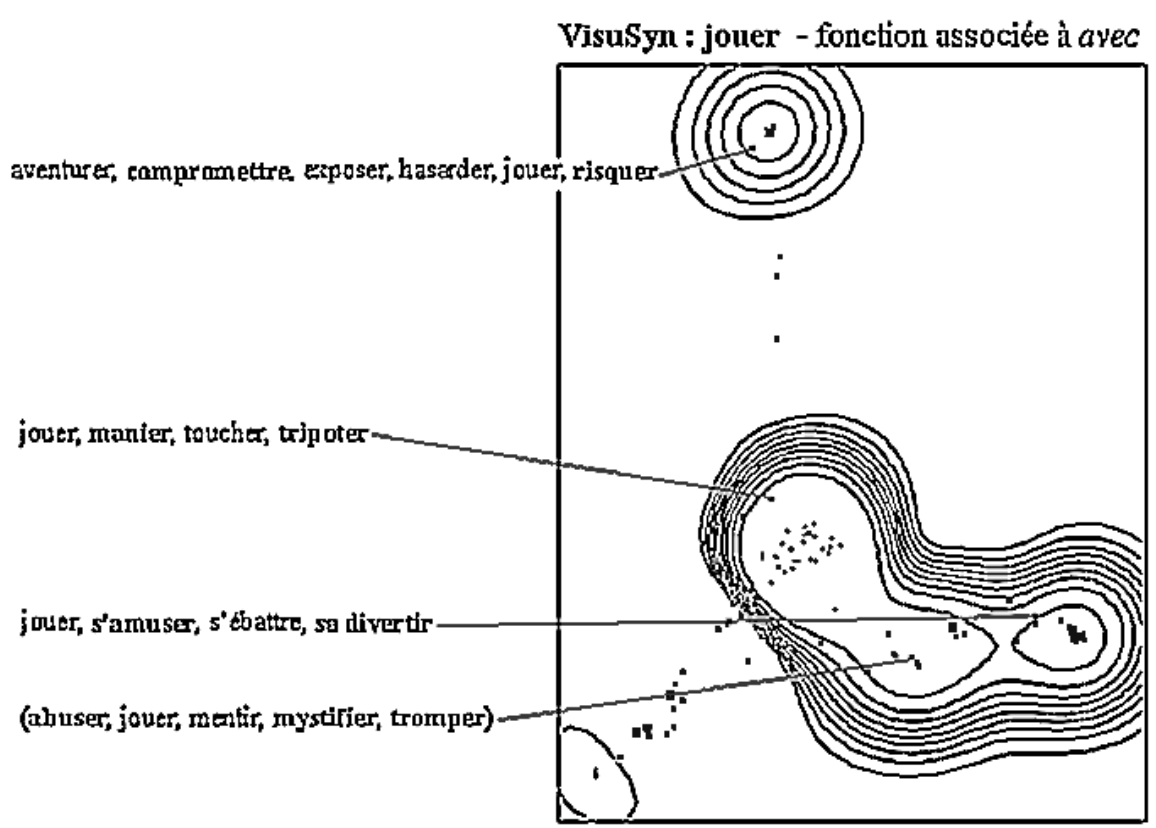

Rigure 3 : espace sémantique de « iouer avee »

Figure 7 extraite de Jacquet (2003)

VisuSyn : jouerSyn - fonction associée à du, de la, (des)

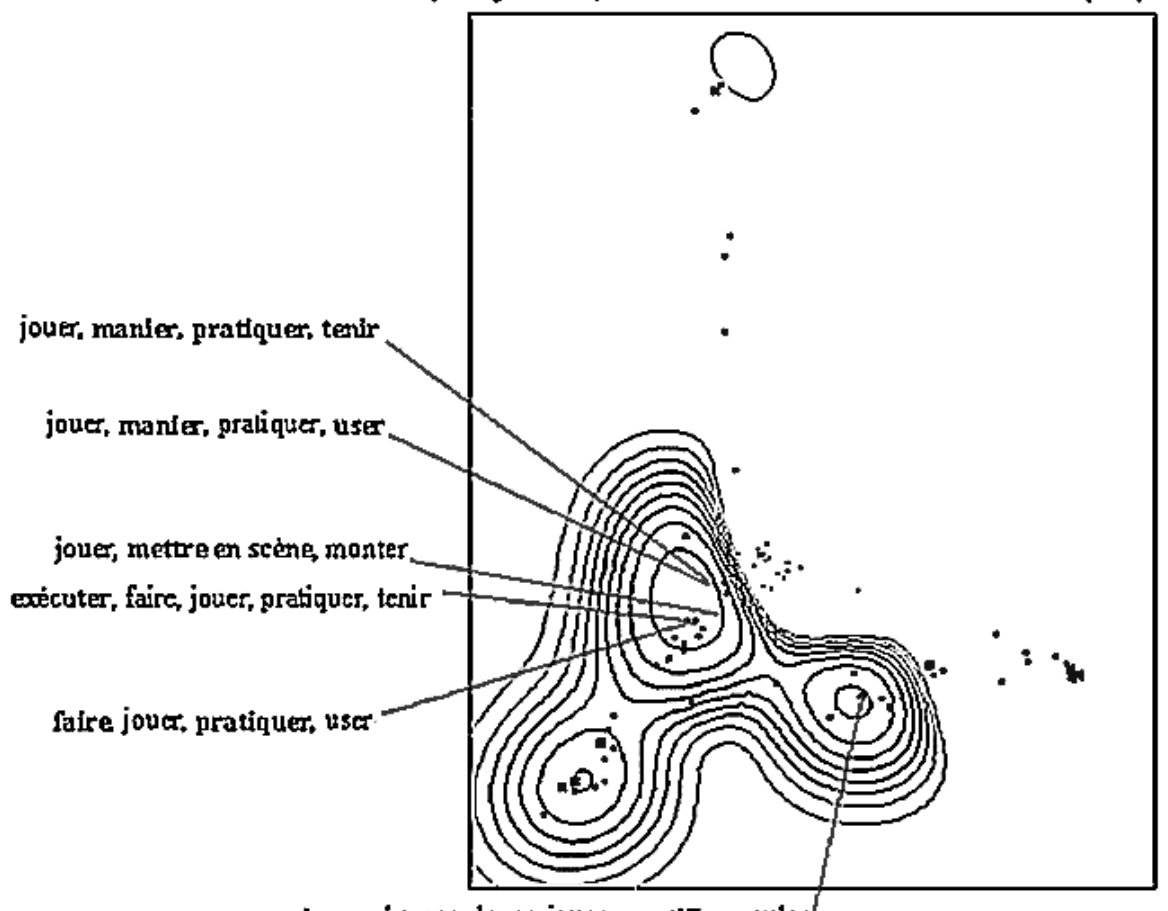

abusa, berner, duper, joster, mystilie, rouler

Figure 8 extraite de Jacquet (2003) 
VisuSyn : jouer - fonction associée à à, au, aux

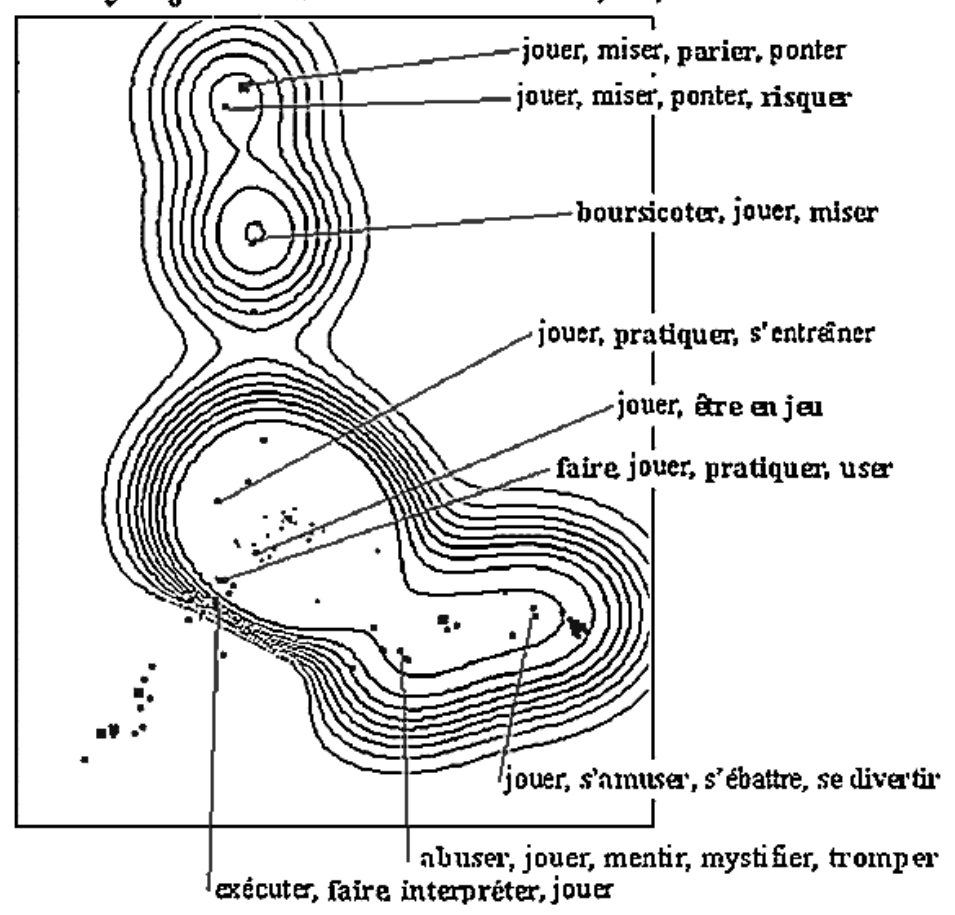

Figure 9 extraite de Jacquet (2003)

\section{Un éventail de points de vue sur la polysémie du verbe monter ${ }^{12}$}

\subsection{Desclés et al. (1998), Desclés \& Guentcheva (2005)}

Desclés \& Guentcheva (2005, p.102) proposent pour le verbe monter un invariant sémantique - dont ils précisent qu'il équivaut à un signifié de puissance dans la tradition de G. Guillaume - «exprimé par abstraction à partir d'un changement et d'un gradient». Dans le domaine spatial, le changement est spécifié comme un mouvement dans l'espace et le gradient est « une différence orientée de hauteur ». En revanche en dehors du domaine spatial, le gradient est soit une différence, soit « de degré dans une échelle orientée du moins vers le plus » soit «d'organisation allant du moins élaboré au plus élaboré ». Et comme le propos de l'article est de comparer la polysémie du verbe monter en français et du verbe (iz)kačvam en bulgare, ils ajoutent que « cette organisation est propre au français ». En effet la notion de croissance organisationnelle est véhiculée en bulgare par un autre verbe (sglobjavam ou montiram emprunté au français).

Auparavant, Desclés \& al. (1998: 43) avaient proposé un diagramme de spécification progressive des différents types d'emploi du verbe monter qui prévoit une distinction primaire entre spécification spatiale et non spatiale.

Dans le domaine conceptuel spatial, deux parcours sont distingués ${ }^{13}$ :

a) un parcours cinématique ( La fumée monte) puis «dynamique avec téléonomie» ( Jean $<$ agent $>$ montait sur la colline) et enfin «dynamique avec transitivité sémantique », c'est-à-dire factitif Luc $<$ Agent + Causateur $>$ monte les oranges $<$ Patient $>$ sur le camion $<$ Locatif-cible $>$ ),

b) un parcours «cinématique virtuel» ( Le chemin<Locatif> montait), puis «dynamique sans téléonomie » $(\checkmark$ Jean $<$ Agent? $>$ montait la colline $<$ Locatif-Patient $>$, qui pose le problème du statut de 
Jean, à proprement parler «humain auto-déplaçant » c'est-à-dire à la fois Agent et Causateur du mouvement de son corps) et enfin «dynamique avec intermédiaire ${ }^{14}$ » $(\checkmark$ Jean $<$ Agent?> montait en ascenseur $<$ Instr $>$, qui pose un problème similaire).

Le domaine conceptuel non spatial est introduit par une opération d'abstraction à chacun de ses niveaux hiérarchiques : générique, cinématique et dynamique. Au niveau cinématique c'est une « variation sur une échelle » qui est exprimée ( Le blé<Entité effectuée/ ?Locatif $>$ monte), au niveau dynamique c'est une «variation dans l'organisation» ( $\$$ Luc $<$ Agent + Causateur $>$ monte un coup $<$ Entité effectuée $>)$. La relation entre Le blé monte et Luc monte un coup est claire du point de vue de l'entité effectuée, à une différence près cependant qui sera soulignée par P. Jalenques (cf. §4.4), c'est que le blé croît au cours du procès à partir d'un degré de développement qui n'est pas nul (la visée est progressive), tandis que le coup vient progressivement à l'esprit de Luc sans que la prédication exprime sa réalisation (ex. Luc a monté un coup qui n'a jamais pu être réalisé), la visée étant donc inchoative. D'autre part l'extension métaphorique du développement d'une entité biologique vers celui d'une entité abstraite n'est examinée en détail dans aucun des deux articles et reste donc mystérieuse.

\subsection{Lebas \& Cadiot (2003)}

L'objectif de F. Lebas et P. Cadiot est d' "illustrer l'idée selon laquelle les objets sont des synthèses d'élaboration par le discours» $(2003: 20)$. Leur illustration porte sur certrains emplois du verbe monter et vise à montrer que «la constitution extrinsèque du référent est en même temps un mode de construction des objets ».

Pour les emplois transitifs de monter, ils partent de l'observation que la «gestalt de la verticalité » se transforme progressivement en gestalt de l'assemblage ou de l'organisation. Pour aboutir à monter une page, un dossier, un kit, un projet, un spectacle, un complot, la métamorphose passe par un stade intermédiaire entre verticalité et assemblage illustré par monter un mur, une mayonnaise (et j'ajouterai monter les æuufs en neige, expression qui fournit à la fois les deux états initial et final de l'opération). Dans tous ces exemples le référent de l'objet direct est effectué au cours du procès.

Pour marquer le lien entre ces emplois et ceux où le référent de l'objet est un lieu, par ex. monter la côte, l'escalier, l'échelle, les gradins, les degrés, les auteurs attribuent une prédicativité faible au verbe « en ce sens que l'objet qu'ils thématisent n'a pas d'extériorité par rapport au procès » (p.25). Selon eux ces substantifs gardent «la trace de leur origine processuelle». C'est clair pour les objets fabriqués pour permettre un déplacement vertical et les lieux aménagés dans le même but. Pour côte, on trouve comme définitions dans le Petit Robert à la fois «II.1. Pente que forme l'un des côtés d'une colline » et «II.2. Route en pente ». Monter la côte sélectionne donc l'acception II.2, c'est suivre une route en pente en s'élevant. Dans la terminologie de Lebas \& Cadiot, on peut dire que monter profile (terme emprunté à R. Langacker) la côte comme une entité « à monter », c'est-à-dire lui attribue une orientation ascendante. Le même raisonnement s'applique à monter un cheval, expression qui profile le cheval « dans son statut de monture, d'entitée programmée pour être montée » (p.25).

Les auteurs concluent que «les référents eux-mêmes sont, en même temps qu'ils s'individualisent, des entités modales investies de pratiques, qu'elles soient inscrites dans leur programmation initiale ou homologuées par le procès actuel». Si le premier cas s'applique à monter un escalier, monter les marches, ainsi qu'à monter un coup, monter une entreprise, le second s'applique à monter la côte. On regrette qu'ils ne mentionnent pas les travaux de J. Pustejovsky (cf. Pustejovsky 1995), car leur notion d'« entités modales investies de pratiques » rappelle la théorie des qualia qui permet de donner un sens à une expression telle que a fast car en présence d'une voiture à l'arrêt, le quale «télique » (c'est-à-dire relatif à la finalité de l'objet) simposant la représentation d'un usage sportif de la voiture.

\subsection{Jalenques (à paraître 1, 2)}

Pierre Jalenques propose dans ces deux articles une analyse critique de l'analyse de la polysémie du verbe monter proposées par Desclés \& al. (1998), Cadiot (1999) et Desclés \& Guentcheva (2005). Il commence 
par critiquer l'invariant sémantique de Desclés \& al. (1998), proche de celui de Desclés \& Guentcheva (2005) :
l'invariant sémantique proposé est trop puissant s'il prédit une distribution de monter trop large par rapport aux données observables. L'invariant de Desclés et al. prédit la compatibilité de monter avec tous les termes pouvant être associés à une gradation orientée vers des valeurs positives : or, c'est loin d'être le cas. De nombreux termes se combinent difficilement avec le verbe monter, alors qu'ils se combinent naturellement avec augmenter (à par.1).

Il donne comme exemples d'incompatibilité avec monter et de compatibilité avec augmenter:

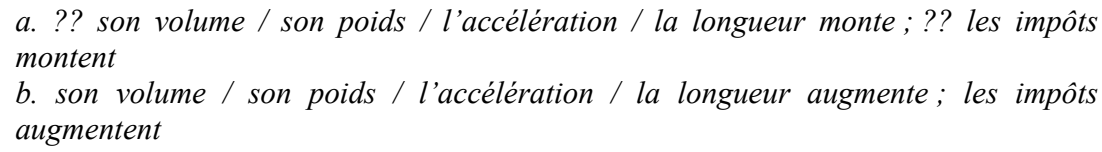

À partir de cette observation, il propose une méthode d'analyse distributionnelle, afin de « trouver un invariant sémantique suffisamment spécifique pour un mot comme monter ». Concrètement, la méthode consiste à examiner en priorité des énoncés « peu naturels» avec le verbe monter pour « mettre au jour la ou les contrainte(s) sémantique(s) de monter en cause dans cet énoncé ». Dans un second temps, il s'agit de déterminer dans quelles conditions contextuelles, et au prix de quelle opération de récupération interprétative, l'énoncé peut cependant être perçu comme naturel.

P. Jalenques observe que Le vent monte est d'un usage moins fréquent que Le vent se lève, mais surtout que se lever et monter en rapport avec le vent ne présupposent pas les mêmes situations initiales : aucun vent n'est perceptible dans le premier cas, contre un vent léger dans le second. Ensuite il examine les sujets possibles pour monter et se lever et constate que seul monter est compatible avec un sujet référant à une grandeur physique : La pression / température monte vs. *se lève $e^{15}$. Concernant la valeur d'assemblage de monter, Jalenques observe que «l'entité qui monte ou bien qui est montée doit déjà avoir un mode de présence du point de vue du domaine sémantique en jeu dans l'énoncé », ce qui rappelle - dans une formulation plus immédiatement compréhensible - la notion d' « entité modale investie de pratiques » proposée par Lebas \& Cadiot (cf. §4.3).

Au final l'auteur considère que « l'identité sémantique invariante d'une unité polysémique comme monter est constituée par la conjonction des contraintes interprétatives que sa présence fait peser sur l'interprétation des énoncés où elle apparaît ». Ce bilan est illustré dans le second article (Jalenques, à paraître 2) par la mise en évidence d'un jeu de contraintes sur la relation entre $\mathrm{V}$ et $\mathrm{N}_{1}$ dans la construction $\left[\mathrm{N}_{0}\right.$ monte $\left.\mathrm{N}_{1<\text { loc }>}\right]$ que résume le tableau 3 ci-après.

Il explique ainsi la disparité entre $\mathrm{N}_{0}$ monte un escalier (colonne de gauche de $\mathrm{T} 3$ ) et ?? $\mathrm{N}_{0}$ monte un escabeau (colonne de droite) :

quand on monte sur un escabeau, ce qui est visé, c'est d'être plus haut qu'au départ en étant sur l'escabeau (et d'ailleurs pas nécesairement dans la partie haute de l'escabeau). Au contraire, on ne monte pas tant des escaliers pour être plus haut / pour être dans la partie haute des escaliers que pour atteindre un autre espace : l'espace du premier étage, par exemple.

et aboutit à un invariant de sens original par rapport aux analyses antérieures de Desclés et Lebas \& Cadiot : « transition sur un domaine D préexistant, conduisant à une nouvelle situation visée, externe à la transition elle-même ». 


\begin{tabular}{|c|c|c|}
\hline acceptable & douteux & inacceptable \\
\hline $\begin{array}{l}\text { Paul a monté les escaliers } \\
\text { en courant. }\end{array}$ & $\begin{array}{l}\text { ? Il monta le talus d'un pas } \\
\text { mal assuré. }\end{array}$ & $\begin{array}{l}\text { ?? Ils ont monté la montagne } \\
\text { en trois heures. }\end{array}$ \\
\hline $\begin{array}{l}\text { Elle monta les marches du } \\
\text { Palais une à une, l'air }\end{array}$ & $\begin{array}{l}\text { ? Elle a monté la colline à } \\
\text { toute vitesse. }\end{array}$ & $\begin{array}{l}\text { ?? Il a monté l'arbre pour se } \\
\text { cacher. }\end{array}$ \\
\hline $\begin{array}{l}\text { pensif. } \\
\text { Il a monté les six étages à } \\
\text { pied. }\end{array}$ & $\begin{array}{l}\text { ? Elle monta rapidement } \\
\text { l'échelle pour prendre un } \\
\text { livre. }\end{array}$ & $\begin{array}{l}\text { ?? Je t'interdis de monter } \\
\text { l'escabeau pour prendre des } \\
\text { bonbons. }\end{array}$ \\
\hline $\begin{array}{l}\text { Sa vieille voiture a du mal à } \\
\text { monter la côte. }\end{array}$ & $\begin{array}{l}\text { ? Sa vieille voiture a du mal à } \\
\text { monter la rue. }\end{array}$ & $\begin{array}{l}\text { ?? Le camion montait la route } \\
\text { à un bon rythme. }\end{array}$ \\
\hline
\end{tabular}

Tableau 3 : Contraintes sur la construction[ $\mathrm{N}_{0}$ monte $\left.\mathrm{N}_{1<l \mathrm{loc}>}\right]$

\subsection{Quelques observations critiques}

Les trois analyses de Desclés, Lebas \& Cadiot et Jalenques partagent un trait commun, à savoir la conception d'un invariant neutre par rapport à la spatialité, c'est-à-dire n'imposant ni ne rejetant un type de représentation dominante relevant du domaine spatial, temporel ou notionnel.

Le point fort de l'analyse de Desclés \& al. (1998) et Desclés \& Guentcheva (2005) est l'élaboration des schèmes sémantico-cognitifs à partir d'opérations sur les archétypes cognitifs statif, cinématique et dynamique. Mais deux points sont sujets à caution: (a) y a-t-il lieu d'orienter les relations entre les schèmes sémantico-cognitifs ? selon quels critères? (b) quelle est la source cognitive de l'extension du schème de transitivité spatiale à l'élaboration d'une organisation supérieure (ex. monter une entreprise) ?

Dans l'analyse de Lebas \& Cadiot (2003), le point fort est l'idée d'une interdépendance entre la prédication verbale et la référence nominale de l'un ou l'autre actant. Mais on ne voit pas bien si cette interdépendance équivaut ou pas à la 'coercion de type' selon Pustejovsky (1995) et on est en droit de se demander ce que signifie exactement (p.29) « constitutivement, tel lieu d'un mouvement qualifié par le mot route, présente les qualités d'une ascension».

Enfin l'analyse de Jalenques (à paraitre 1,2) apporte du nouveau avec sa méthode d'exploitation des jugements sur la naturalité des paires \{verbe, cadre prédicatif\}, mais à propos de l'exemple Sa vieille voiture a du mal à monter la côte, on s'interroge sur le sens exact de l'assertion : «le terme côte réfère non pas à un chemin mais à une transition sur un chemin ».

Au regard de ces différentes approches, je voudrais formuler une hypothèse à propos de la structuration sémantico-cognitive des emplois transitifs à objet non locatif du verbe monter : j'y vois le résultat de la combinaison de deux dérivations $\{+$ concret $\Rightarrow$-concret\} et \{+élémentaire $\Rightarrow$-élémentaire $\}$. Dans la première classe $\{+$ concret / +élémentaire $\}$ on range aussi bien les emplois généraux de déplacement causatif (monter un objet matériel qqpart) que l'emploi spécifique d'enchâssement (monter une pierre sur une bague). On en dérive d'une part une classe par abstraction de l'objet (monter le son, monter la pression) et d'autre part une autre classe par complexification de l'opération (monter un moteur, c'est-àdire assembler les pièces du moteur, monter un film, c'est-à-dire assembler les scènes du film par découpage et collage matériel des fragments du film). Enfin une quatrième classe \{-concret / élémentaire $\}$ résulte de la composition de l'opération d'abstraction et de celle de complexification de l'objet, donnant monter une entreprise, un spectacle ou un coup, cf. Tableau $4^{16}$. 


\begin{tabular}{|c|c|c|}
\hline & + concret --- & $-->$-concret \\
\hline +élémentaire & $\begin{array}{l}\text { Le porteur monte la malle dans le train vs. } \\
\text { descend /*démonte la malle du train. } \\
\text { Le bijoutier monte une pierre précieuse } \\
\text { sur une bague vs. détache /*démonte la } \\
\text { pierre de la bague }\end{array}$ & $\begin{array}{l}\text { Marie monte le son pour écouter } \\
\text { les informations vs. baisse le son. } \\
\text { Le chauffeur de la locomotive } \\
\text { monte la pression vs. baisse la } \\
\text { pression. }\end{array}$ \\
\hline $\begin{array}{c}\downarrow \\
\text {-élémentaire }\end{array}$ & $\begin{array}{l}\text { Le mécanicien monte le moteur / les } \\
\text { pièces du moteur vs. démonte le moteur / } \\
\text { les pièces } \\
\text { Le monteur monte le film / les scènes du } \\
\text { film vs.??? }\end{array}$ & $\begin{array}{l}\text { Le truand monte une affaire } \\
\text { louche vs.ø } \\
\text { Le metteur en scène monte } \\
\text { Roméo \& Juliette vs. } \varnothing\end{array}$ \\
\hline
\end{tabular}

Tableau 4 : La combinaison des deux dérivations $\{+\Rightarrow$-concret $\}$ et $\{+\Rightarrow$-élémentaire $\}$

Cette proposition est explicitement dérivationnelle. Mais on peut en imaginer une variante dans la perspective de Lebas \& Cadiot (2003) si l'on admet, comme ils le font, que le référent de l'objet porte en lui-même ou acquiert par combinaison avec le verbe un profil compatible avec la notion abstraite de progression entre deux niveaux. La malle est «destinée» dans la pratique du porteur à passer du quai dans le train, la pierre est « destinée » dans celle du joailler à surmonter une bague, le son et la pression sont destinés à varier sur une échelle d'intensité, les pièces du moteur et les scènes du film sont organiquement destinées à constituer un moteur ou un film. Enfin une entreprise et un spectacle sont par essence des objets abstraits et complexes qui n'existent initialement qu'à l'état de représentation mentale d'un agent (réalisateur, entrepreneur ou metteur en scène) et qui viennent au jour par un ensemble complexe d'opérations de réalisation factuelle.

\section{La 'colexicalisation' du verbe monter : un montage et démontage perpétuel}

Alexandre François a proposé récemment (2008:170) une théorie de la typologie lexicale combinant les deux notions de «carte sémantique» selon le modèle initié par Haspelmath (2003) et de « colexicalisation » qu'il applique à la comparaison lexicale intralangue et interlangue :

Once a list of senses is arrived at, the phenomenon most relevant for the second stage of observation may be called COLEXIFICATION.

(1) "A given language is said to COLEXIFY two functionally distinct senses if, and only if, it can associate them with the same lexical form $1 . "$

[...] One of the advantages of the term "colexification", which I am proposing here, is to be purely descriptive, and neutral with respect to semantic or historical interpretations - contrary to the term "semantic shift" [...]

L'affirmation de la neutralité et du caractère strictement descriptif de la notion de colexicalisation n'est cependant pas sans poser problème. En effet A. François illustre cette théorie par l'examen typologique des expressions nominales et verbales du souffle respiratoire qui donne lieu à l'élaboration d'une carte sémantique de la colexicalisation des sens concrets et abstraits des termes de souffle à travers la variété des langues prises en compte. La comparaison des cartes sémantiques est particulièrement instructive pour une même langue, par exemple celles de anima et spiritus en latin, et de $\pi v \varepsilon v \mu \alpha$ et $\pi \sigma v \chi \eta$ en grec classique. Le fait de concevoir comme tertium comparationis une carte représentant l'union de cartes lexicales particulières impose - en tout cas c'est le cas pour son illustration - un «pivot» sémantique commun (cf. Figure 13) : 


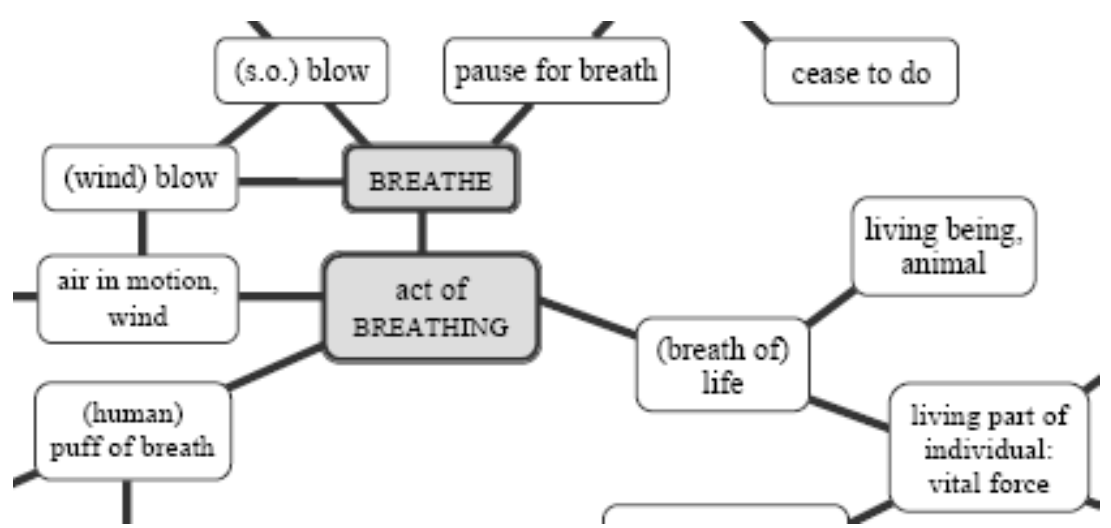

Figure 13: Le pivot immédiat de la carte sémantique de BREATHE / act of BREATHING et sa périphérie immédiate selon A. François (2008:202)

Selon l'auteur (2008:182) ce pivot n'est rien de plus que le point de départ de la construction de la carte sémantique $^{17}$, mais le fait de rassembler dans une même étude les termes anglais breathe, sanscrit ätman, grec $\pi v \varepsilon v \mu \alpha / \pi \sigma v \chi \eta$, latin anima / spiritus, etc. implique que ces termes comportent RESPIRATION comme une composante essentielle de leur champ sémasiologique et de fait tous les items examinés partagent cette représentation sémantique comme pivot de leur polysémie. On est donc loin de la neutralité effective de l'approche cartographique présentée précédemment dans les sections 3 et 4.3, et le mode de représentation qu'illustre la figure 13 est étroitement apparenté à celui que privilégient Fillmore \& Atkins (cf. figure 1) à ceci près que ces derniers ne cherchent pas à unifier les deux réseaux sémantiques de nager et to crawl en un réseau interlexical (et interlangue).

A. François $(2008: 171)$ ajoute que cette théorie est également applicable à la comparaison de deux états de langue :

Strictly speaking, the notion of colexification should be understood as "the capacity, for two senses, to be lexified by the same lexeme in synchrony". However, nothing prevents the model from being extended, so as to make provision for several hierarchized ? - levels of colexification. These may include the linking of two senses by a single lexeme across different periods of its semantic history $[\ldots]$

C'est dans cette perspective que je vais étudier, en matière de conclusion, comment s'est fait, défait et refait au cours des siècles le champ sémasiologique du verbe monter. Je partirai de la synthèse des acquis du FRANZÖSISCHES ETYMOLOGISCHES WÖRTERBUCH que présente la composante Histoire et Étymologie de l'article monter dans le TRESOR DE LA LANGUE FRANÇAISE (version informatisée). Cette composante est extrêmement détaillée avec 51 rubriques. Un premier niveau de classement distingue les emplois intransitifs (I) des emplois transitifs (II), puis un second niveau distingue parmi les emplois intransitifs ceux à sujet animé (I.A) et ceux à sujet non-animé (I.B). Dans les tableaux 5 (emplois intransitifs) et 6 (emplois transitifs) j'indique par un « $1 » l^{\prime}$ 'attestation de chaque emploi du $10^{\mathrm{e}}$ au $20^{\mathrm{e}}$ siècle ou l'absence de mention de son extinction. Les données du TLIi permettent de fixer précisément l'innovation sémantique. En revanche, s'agissant des emplois qui ne sont plus attestés au $20^{\mathrm{e}}$ siècle, la fixation de l'époque d'extinction sémantique est beaucoup plus délicate.

Concernant le tableau 5, pour l'emploi I.A.4.a : monter en ire « se mettre en colère », daté de 1174, je n'ai pas d'élément pour juger de la persistance de cette construction au-delà de l'ancien français. Pour l'emploi I.B.2b on peut supposer que la construction datée de 1178 : monter en la teste « enivrer (en parlant du vin) », a été rapidement remplacée par la construction monter à la tête (même rubrique) datée de 1668. 


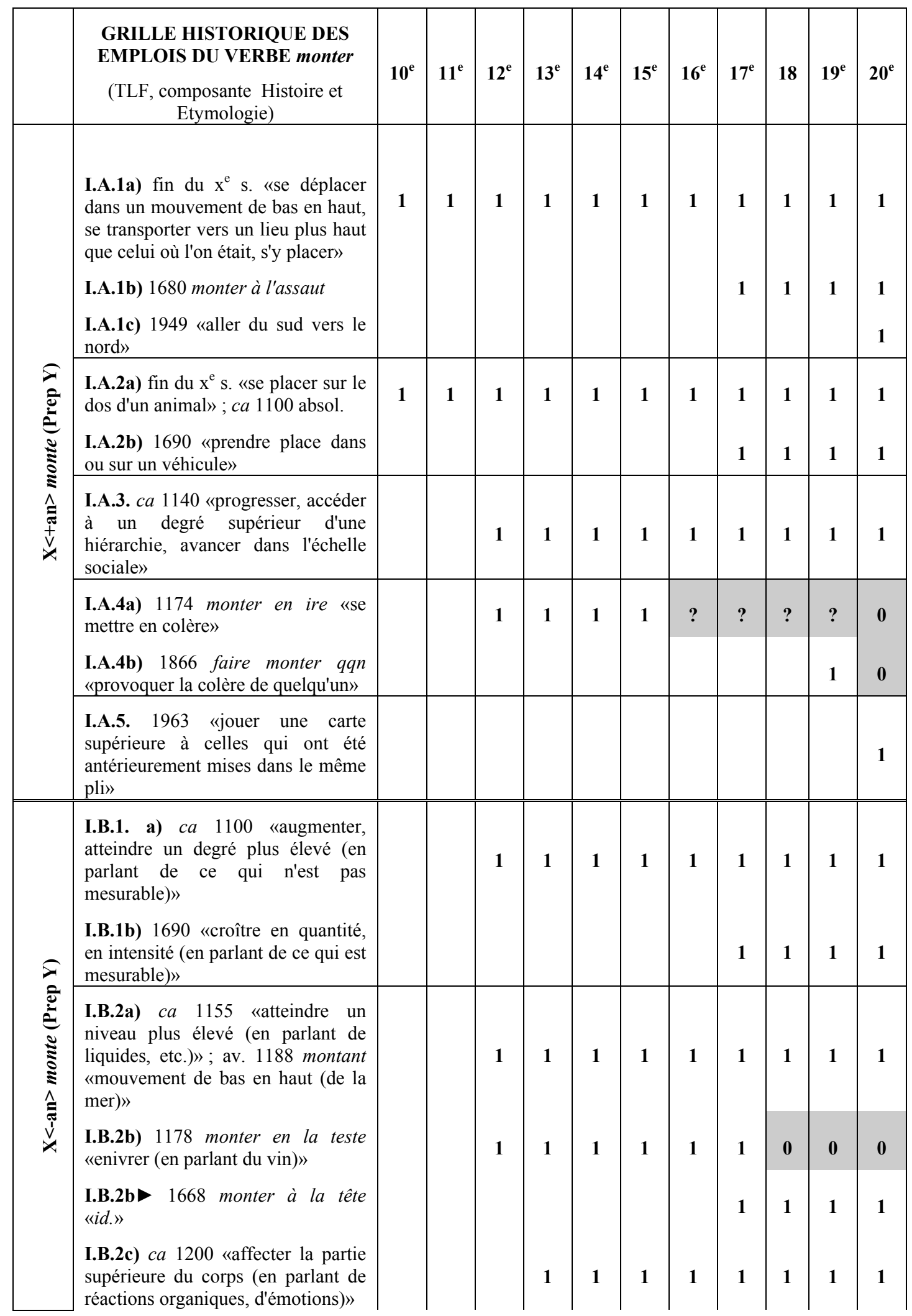




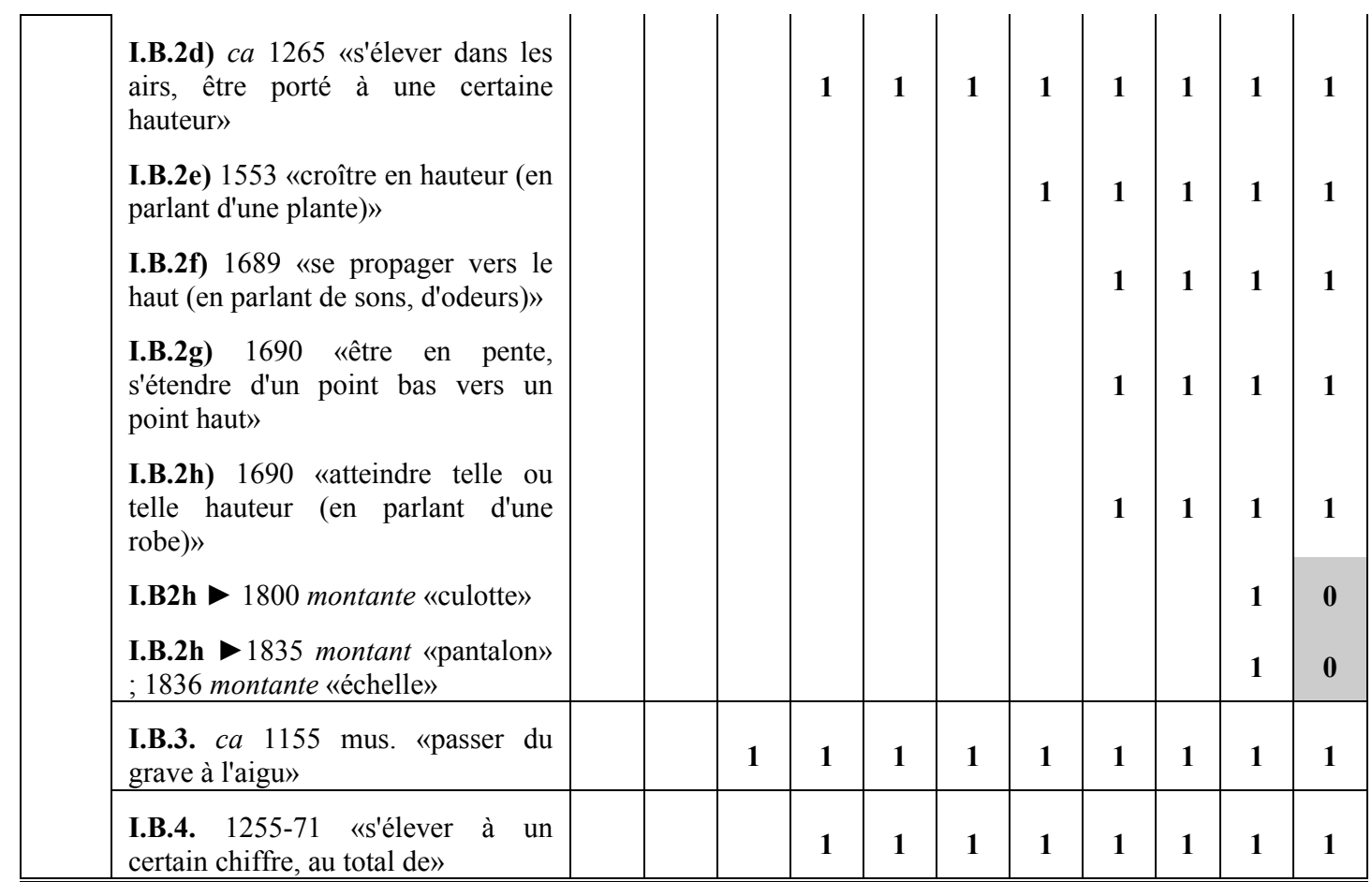

Tableau 5 : La classe I de la composante Histoire et Étymologie de l'article monter du TLFi

Concernant le tableau 6, seule une analyse longitudinale de la base FRANTEXT permettrait de déterminer à partir de quelle époque l'emploi II.2c daté de 1174 : monter qqn " accroître la valeur (de quelqu'un) » est sorti d'usage. Le DICTIONNAIRE DU MOYEN-FRANÇAIS ${ }^{18}$ fournit trois attestations de cet emploi en 1387, 1400 et 1456. En revanche le THRESOR de Nicot (1610) ni aucune des éditions du DICTIONNAIRE DE L'ACADEMIE ne la mentionnent. On peut donc supposer qu'elle a disparu à partir du $16^{\mathrm{e}}$ ou $17^{\mathrm{e}}$ siècle. Particulièrement intrigante est la construction monter un navire «servir comme marin sur (un navire)» datée de 1680 (rubrique II.5b) car elle est bien attestée dans le dictionnaire de Furetière (1690) et la $1^{\text {ère }}(1694)$, la $4^{\mathrm{e}}(1798)$, la $6^{\mathrm{e}}(1832)$ et la $8^{\mathrm{e}}(1932)$ éditions du DICTIONNAIRE DE

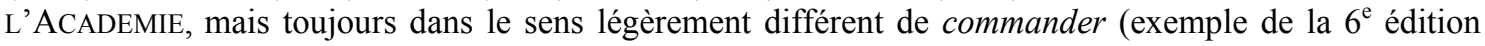
repris dans la $8^{\mathrm{e}}$ : Le contre-amiral montait le vaisseau le Formidable).

Compte tenu du nombre important de rubriques répertoriées par le TLFi, je ne chercherai pas à représenter les avatars de la colexicalisation du verbe monter sous la forme d'une carte sémantique. Je me contenterai de comparer à titre d'illustration le sous-ensemble des rubriques des tableaux 5 et 6 qui concernent respectivement les $10^{\mathrm{e}} / 11^{\mathrm{e}}$ siècles et les $12^{\mathrm{e}} / 13^{\mathrm{e}}$ siècle parce qu'une multitude d'innovations se sont fait jour entre ces deux époques. La carte sémantique de la page suivante représente la colexicalisation du verbe monter atteinte au $13^{\mathrm{e}}$ siècle et elle spécifie par deux encadrés la colexicalisation très limitée des $10^{\mathrm{e}} / 11^{\mathrm{e}}$ siècles et celle déjà très étendue du $12^{\mathrm{e}}$ siècle. Les deux emplois entourés d'une ellipse ont disparu au $20^{\mathrm{e}}$ siècle, monter qqn $\approx$ accroître la valeur de qqn mentionné plus haut et monter qqn sur une monture plutôt remplacé ${ }^{19}$ par faire monter qqn sur une monture.

Il serait naturellement instructif de comparer l'explosion de la polysémie du verbe monter autour du $12^{\mathrm{e}}$ siècle avec la variété aux mêmes époques des emplois attestés des verbes du même champ sémantique des mouvements orientés : gravir, descendre, choir, franchir, entrer, sortir et surtout passer. 


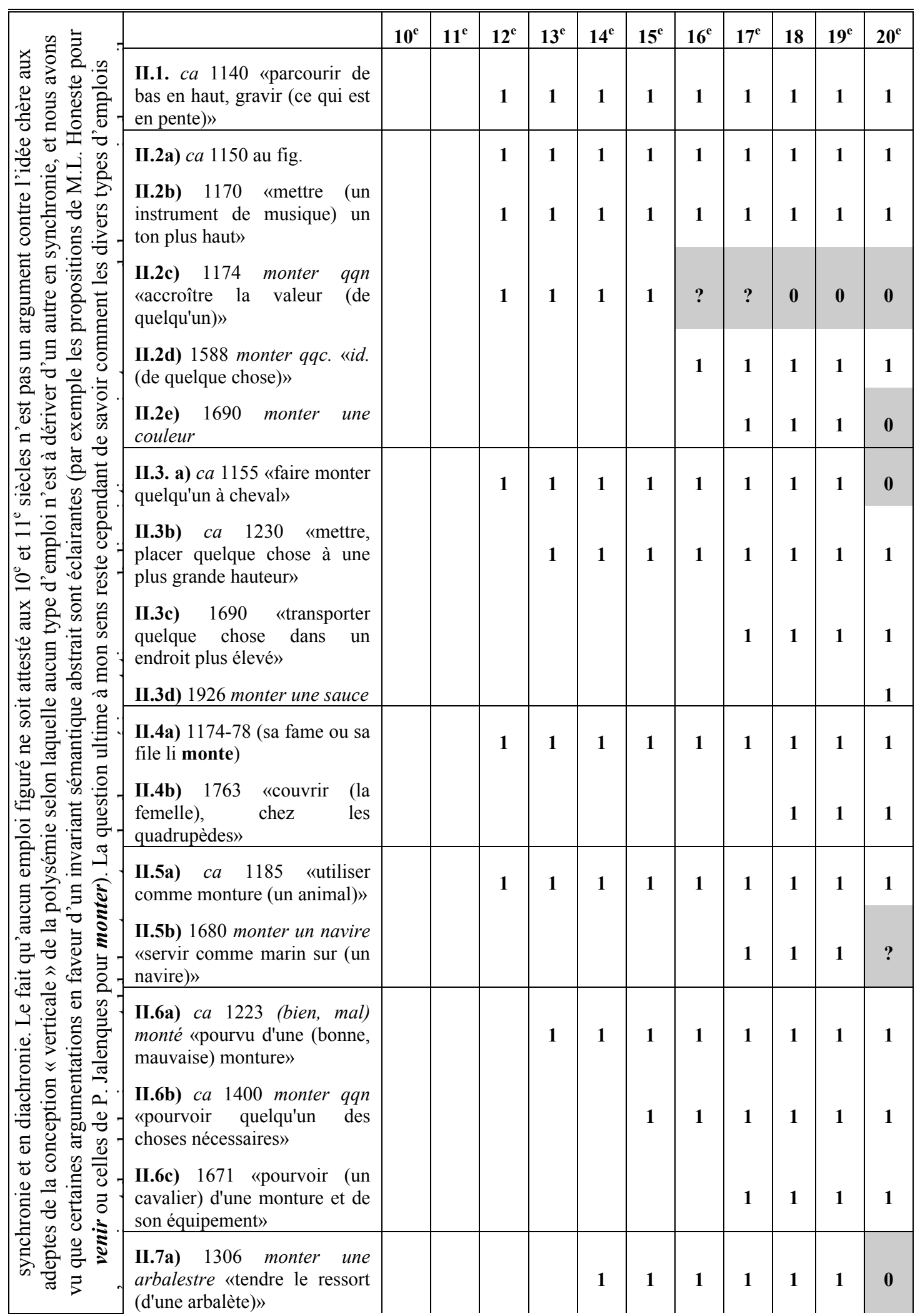




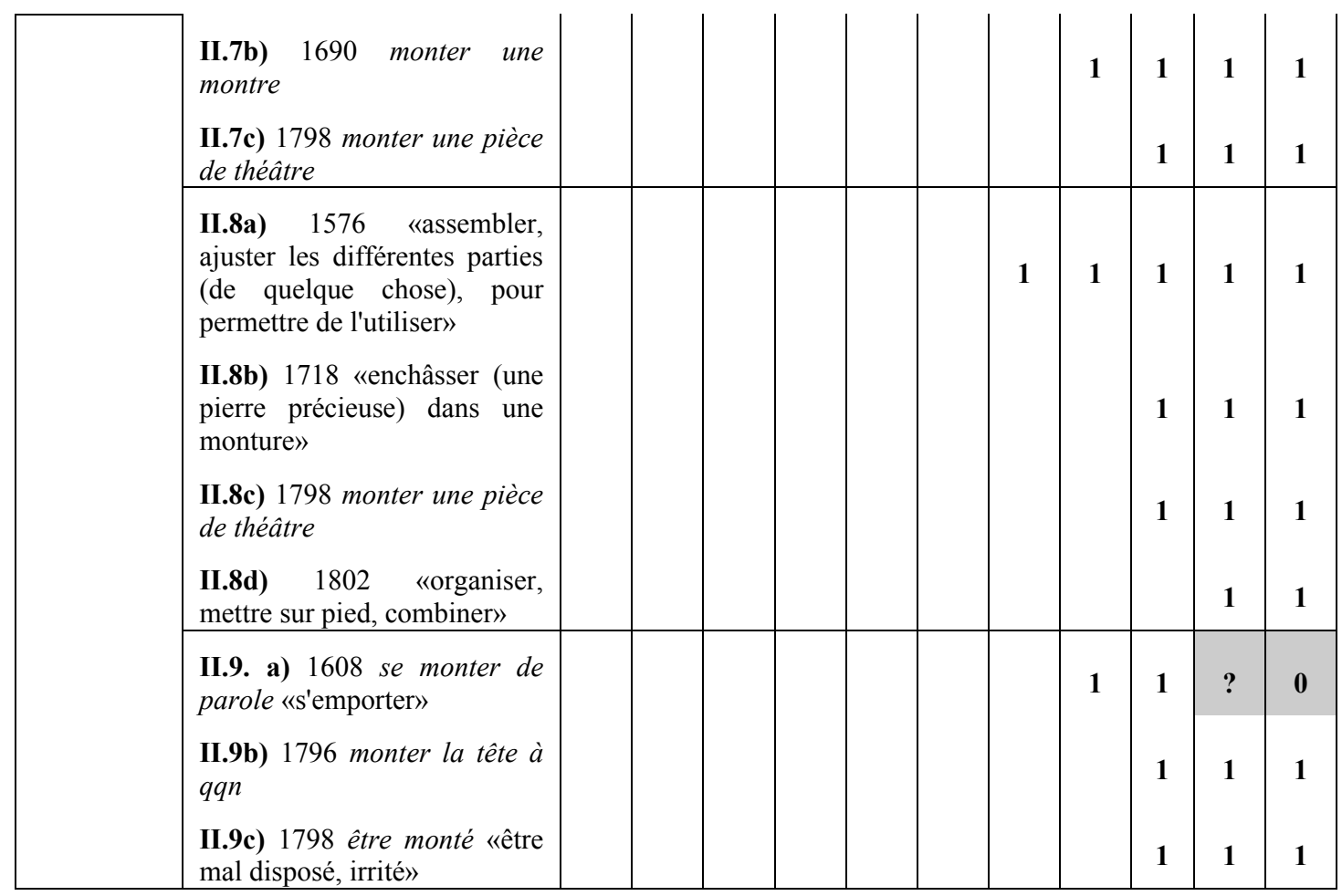

Tableau 6 : La classe II de la composante Histoire et Étymologie de l'article monter du TLFi 


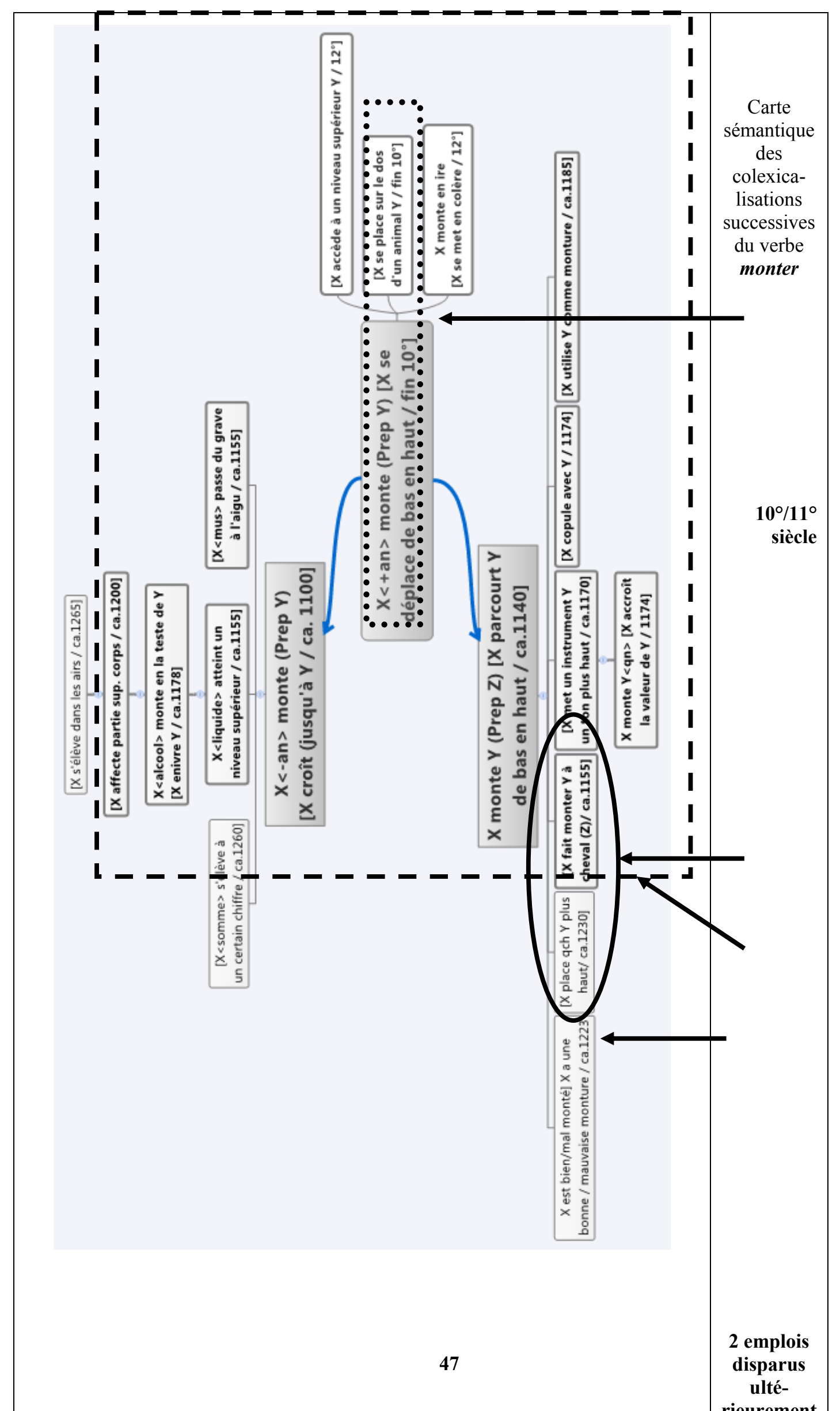




\section{Références principales}

Desclés J.P. \& al. (1998), Sémantique cognitive de l'action : 1. contexte théorique. Langages 132, p.28-47

Desclés, J.P. (2005a), Polysémie verbale. Conférence à l'université de Varsovie, 4.4.2005.

Desclés, J.P. \& Guentcheva, Z. (2005), Doit-on tenir compte de la polysémie verbale en typologie ? Un exemple contrastif entre français et bulgare. Langue Française 145, p.93-107

Fillmore, Ch. \& Atkins, M. (2000), Describing polysemy : the case of 'crawl'. In : Y. Ravin \& C. Leacock (eds, 2000), Polysemy - Theoretical and computational approaches. Oxford : Oxford University Press, p. 91-110.

Franckel, J.J. (2002), A propos du rôle des unités morpho-lexicales dans la construction du sens des énoncés. In : D. Lagorgette \& P. Larrivée (dir.), Représentations du sens linguistique. Munich : LINCOM Europa, p.255-265.

Franckel, J.J. (2004), Sentir / sens. In : Camus, R \& de Voguë, S. (dir. 2004), Variation sémantique et syntaxique des unités lexicales : étude de six verbes français. LINX n50. Université Paris-X Nanterre. p.103-134.

François, A. (2008), Semantic maps and the typology of colexification: Intertwining polysemous networks across languages. In : M. Vanhove (ed.), From polysemy to semantic change. Amsterdam et al. : Benjamins, p.163-216.

François, J. (2007a), Cartographie de la polysémie verbale. Bibliothèque de la Société de Linguistique de Paris. Louvain : Peeters.

François, J. (2007b), L'espace sémantique des verbes fr. tenir et alld halten : noyau et extensions. In C. Paulin \& al. (dir.), Constructions verbales et production du sens. Actes $d u$ Colloque de Besançon, 26-28 janvier 2006. Presses Universitaires de Franche-Comté, p.293-306.

François, J. (2007c), Combining a semantic map and a dual text corpus analysis for analysing verb polysemy (illustrated by french jouer). In A.Sellami-Bakouti \& M. Triki (eds.), Ambiguity and Disambiguation. University of Sfax, GRAD, p.13-30.

François, J. (à paraître) Quand jouer, c'est jouer de la musique : Repérage contextuel de quatre zones de l'espace sémantique du verbe jouer. In : E. Richard (dir.), Actes du colloque en hommage à M. Noailly, Rennes 2005. Presses Universitaires de Rennes.

Honeste, M.L. (2005a), La théorie des schémas conceptuels intégrés : un prolongement de la théorie guillaumienne ? Langue Française 147, p.68-83

Honeste, M.L. (2005b), Venir est-il un verbe périphrastique ? Etude sémantico-cognitive. In H. Bat-Zeev Shyldkrot \& N. le Querler (dir.), Les périphrases verbales. Amsterdam : Benjamins, p. 293-309.

Jacquet, G. (2003), Polysémie verbale et construction syntaxique : étude sur le verbe jouer. Actes du colloque TALN 2003, Batz-sur-Mer, 11-14 juin 2003.

Jacquet, G. (2004), Polysémie verbale et calcul du sens. Thèse de doctorat de l'EHESS / Lattice CNRS.

Jalenques, P. (à paraître 1), Analyse sémantique et contraintes distributionnelles : l'exemple du verbe monter. Actes du CILPR 2007, Innsbruck.

Jalenques, P. (à paraître 2), Valeurs spatiales et valeurs non spatiales dans le domaine des verbes: pour une sémantique constructiviste. Actes du $5^{\mathrm{e}}$ colloque de la revue Sémantique et pragmatique.

Lebas, F. \& Cadiot, P. (2003), Monter et la constitution extrinsèque du référent. Langages 150, p.9-30

\section{Bibliographie complémentaire}

Abraham, M., (2005), Représentation et structuration de la polysémie verbale - un exemple . In Soutet (dir.), p.137154

Bat-Zeev Schylkrot, H., (1997) La grammaticalisation des auxiliaires : le cas de voir. SCOLIA 10, pp.205-224. Université Marc-Bloch Strasbourg 2.

Cadiot, P.., (1999) Les sens de jouer : esquisse d'une approche par le biais des attaches prépositionnelles. Recherches en linguistique et psychologie cognitive $\mathrm{n}^{\circ} 11$. Presses Universitaires de Reims

Camus, R., (2004, Quelques aspects de commencer. In Camus \& de Voguë (dir. p. 81-102). 
Camus, R. \& de Voguë (dir. p. 81-102)., Variation sémantique et syntaxique des unités lexicales : étude de six verbes français. LINX n50. Université Paris-X Nanterre.

de Voguë, S. (2004), Syntaxe, référence et identité du verbe filer. In Camus \& de Voguë (dir. 2004), p.135-167.

de Voguë, S. \& D. Paillard (1997), «Identité lexicale et hétérogénéité de la variation co-textuelle : le cas de suivre . In : C. Guimier (dir. 1997), Co-texte et calcul du sens. Caen : Presses Universitaires de Caen, p.41-61.

Desclés, J.P., (2005b), Polysémie verbale, un exemple : le verbe 'avancer'. In : O. Soutet (dir.), La polysémie. Presses Universitaires de Paris-Sorbonne ; p. 111-136.

Desclés, J.P. \& Guentcheva, Z. (2005), Doit-on tenir compte de la polysémie verbale en typologie ? Un exemple contrastif entre français et bulgare. Langue Française 145, p.93-107

Desclés, J.P., Falgeul, V. , Kekenbosch, C., Meunier, J.M. \& Richard, J.F., (1998) Sémantique cognitive de l'action : 1. contexte théorique. Langages 132, p.28-47

Dutoit, D. \& François, J. (2007), Changer et ses synonymes majeurs entre syntaxe et sémantique : le classement des VERBES FRANÇAIS en perspective. In : J. François, D. Le Pesant \& D. Leeman (dir.) Classes syntactico-sémantiques et polysémie. Langue Française 153. p.40-57.

Fall, K. \& Bétoté Akwa, D., (2002), Énonciation et forme du sens - Identité lexicale et variations sémantiques des mots MANGER, AIMER et RAISON. Presses de l'Université Laval / Presses de l'Université de Limoges.

Fillmore, Ch. \& S. Atkins, (2000), Describing polysemy : the case of 'crawl' ». In : Y. Ravin \& C. Leacock (eds, 2000), Polysemy - Theoretical and computational approaches. Oxford : Oxford University Press, p. 91-110.

Franckel, J.J., (2005), Continu/discontinu en sémantique lexicale. L'exemple du verbe changer. Cahiers de praxématique $\mathrm{n}^{\circ} 42$, (D. Ablati \& M. Valette, dir.), Du continu : son et sens.

Franckel, J.J. \& Lebaud, D., (1990), Les figures du sujet - A propos des verbes de perception, sentiment, connaissance. Gap : Ophrys

Franckel, J.J., Paillard, D. \& Saunier E., (1997), Modes de régulation et la variation sémantique d'une unité lexicale. Le cas du verbe passer. In : P. Fiala, P. Lafon \& M.F. Piguet (dir. 1997), La locution : entre lexique, syntaxe et pragmatique - Identification en corpus, traitement, apprentissage. Paris : Klincksieck, p.49-68.

François, J., (1996), Semantische und syntaktische Gliederungskriterien in der Verbpolysemie - Entwurf eines Kalkülverfahrens am Beispiel von frz. affecter. In : E. Weigand \& F. Hunsnurscher (eds. 1996), Lexical structures and language use, volume 2 : Session Papers. Tübingen: Niemeyer, p.53-64.

François, J., (2000), Désémantisation verbale et grammaticalisation : (se) voir employé comme outil de redistribution des actants. In : Cordier, François \& Victorri (dir. 2000), p.159-175

François, J., (2008), The French verb AFFECTER in three types of news articles : a geometric representation with semantactic areas. In S. Heiden \& B. Pincemin (dir.), JADT 2008 : 9èmes Journées internationales d'Analyse des Données Textuelles.

François, J. (2009), Fléchage synonymique ou analyse componentielle dans l'examen de la polysémie verbale? affecter vs. compter, Pratiques $\mathrm{n}^{\circ} 141-142$, p.65-78

Gayral, F., (2000), Traitement automatique et polysémie des verbes. In Cordier, François \& Victorri (dir. 2000), p. $241-25$

Haspelmath, M. (2003), The geometry of grammatical meaning : semantic maps and cross-linguistic comparison. In M. Tomasello (ed.), The new psychology og language, Volume 2. New-York : Erlbaum, 211-243.

Jackendoff, R. (1983), Semantics and Cognition. Cambridge (Masss.) : MIT Press

Jackendoff, R. (1900), Semantic Structures. Cambrindge (Mass.): MIT Press

Jackendoff, R. (2002), Foundations of Language - Brain, Meaning, Grammar, Evolution. Oxford, New York : Oxford University Press

Jacquet, G., Venant, F. \& Victorri, B. (2005), Polysémie lexicale,in P. Enjalbert (dir.), Sémantique et traitement automatique du langage naturel. Lavoisier / Hermès Science Publications, 99-132. 
Lebaud, D., (2004) Toucher : le tango des sens. Problèmes de sémantique lexicale. In : R. Camus \& S. de Voguë (dir., p.53-80).

Lehman, A. \& M. Martin-Berthet., (1998), Introduction à la lexicologie : Sémantique et morphologie. Paris : Dunod

Manguin, J.-L. \& François, J. (dir. 2004), Le dictionnaire électornique des synonymes du CRISCO - un mode d'emploi à trois niveaux. Cahier 17 du CRISCO (www.crisco.unicaen.fr)

Paillard, D. (2000), A propos des verbes 'polysémiques' : identité sémantique et principes de variation. In : Cordier, François \& Victorri (dir. 2000), p. 99-120.

Picoche, J., (1986), Les structures sémantique du lexique français. Paris : Nathan-Université

Picoche, J., (1994), A 'continuous definition' of polysemous items: its basis, resources and limits. In : C. Fuchs et B. Victorri (eds. 1994), Continuity in linguistic semantics. Amsterdam : Benjamins. 77-92.

Pottier, B., (1987), Théorie et analyse en linguistique. Paris : Hachette

Pustejovsky, J. (1995), The generative lexicon. Cambridge (Mass.): MIT Press

Romero-Lopez M.-Ch. (2002), Identité et variété du verbe jouer. Langue Française 133, 63-73.

Soutet, O. (dir. 2005), La polysémie. Paris : Presses Universitaires de Paris-Sorbonne.

Thuillier, F., (2004), Le verbe paraître: surgissement, manifestation, impression, ouï-dire. In Camus \& de Voguë (dir. 2004), 15-32.

Victorri, B. \& Fuchs, C. (1996), La polysémie - Construction dynamique du sens. Paris : Hermès.

Willems, D., (2002), La lexicographie à l'épreuve de la syntaxe et de l'usage : le cas du verbe charger. In I. Bartning, J. Falk \& alii (eds.), Mélanges publiés en hommage à Gunnel Engwall. Acta Universitatis Stockholmiensis 20, Almqvist \& Wiksell International, Stockholm, pp.343-350.

\section{Annexe}

\section{Classement par verbe des travaux sur la polysémie verbale} publiés à partir de $1986^{20}$

\begin{tabular}{|c|c|c|c|c|c|}
\hline Verbe & Auteur & Date & Verbe & Auteur & Date \\
\hline \multirow[t]{2}{*}{ affecter } & FRANÇOIS, J. & 1996 & & LEBAS F. \& CADIOT P. & 2003 \\
\hline & FRANÇOIS, J. & 2009 & & JACQUET, G. & 2004 \\
\hline aimer & PICOCHE, J. & 1986 & & $\begin{array}{l}\text { MANGUIN } \quad \text { J.L. } \quad \& \\
\text { FRANÇOIS, J. (analyse de J.L. } \\
\text { Manguin) }\end{array}$ & 2004 \\
\hline arriver & DESCLÉS, J.P. & 2005 & & $\begin{array}{l}\text { DESCLÉS, } \quad \text { J.P. } \quad \text { \& } \\
\text { GUENTCHEVA, Z. }\end{array}$ & 2005 \\
\hline avancer & DESCLÉS, J.P. & 2004 & & JALENQUES Pierre & à par. \\
\hline \multirow[t]{4}{*}{ changer } & PICOCHE, J. & 1986 & paraître & THUILLIER, F & 2004 \\
\hline & POTTIER, B. & 1987 & passer & $\begin{array}{l}\text { FRANCKEL, J.J., PAILLARD, } \\
\text { D. \& SAUNIER, E. }\end{array}$ & 1997 \\
\hline & FRANCKEL, J.J. & 2005 & pousser & ABRAHAM, M. & 2004 \\
\hline & $\begin{array}{l}\text { DUTOIT, } \quad \text { D. } \quad \& \\
\text { FRANÇOIS, J. }\end{array}$ & 2007 & pouvoir & GOSSELIN, L. (p.439-55) & 1010 \\
\hline
\end{tabular}




\begin{tabular}{|c|c|c|c|c|c|}
\hline charger & WILLEMS, D. & 2002 & prendre & PAILLARD, D. & 2000 \\
\hline commencer & CAMUS, R. & 2004 & ramper & $\begin{array}{l}\text { FILLMORE, Ch. \& ATKINS, } \\
\text { S. }\end{array}$ & 2000 \\
\hline \multirow[t]{3}{*}{ compter } & PAILLARD, D. & 2000 & relever & $\begin{array}{l}\text { LEHMANN, A. \& MARTIN- } \\
\text { BERTHET, M. }\end{array}$ & 1998 \\
\hline & JACQUET, G. & 2005 & rendre & $\begin{array}{l}\text { FRANCKEL, J.J. \& LEBAUD, } \\
\text { D. (p.178-190) }\end{array}$ & 1990 \\
\hline & FRANÇOIS, J. & 2009 & respirer & PICOCHE, J. & 1986 \\
\hline connaître & $\begin{array}{l}\text { FRANCKEL, J.J. \& } \\
\text { LEBAUD, D. (p.103-116) }\end{array}$ & 1990 & savoir & $\begin{array}{l}\text { FRANCKEL, J.J. \& LEBAUD, } \\
\text { D. (p.90-102) }\end{array}$ & 1990 \\
\hline couper & GAYRAL, G. & 2000 & sentir & FRANCKEL, J.J. & $\begin{array}{l}2002, \\
2004\end{array}$ \\
\hline craindre & $\begin{array}{l}\text { FRANCKEL, J.J. \& } \\
\text { LEBAUD, D. (p.147-156) }\end{array}$ & 1990 & souhaiter & $\begin{array}{l}\text { FRANCKEL, J.J. \& LEBAUD, } \\
\text { D. (p.119-124) }\end{array}$ & 1990 \\
\hline défendre & PICOCHE, J. & 1986 & suivere & $\begin{array}{l}\text { DE VOGUË, } \\
\text { PAILLARD, } \text { D. }\end{array}$ & 1997 \\
\hline désirer & $\begin{array}{l}\text { FRANCKEL, J.J. \& } \\
\text { LEBAUD, D. (p.125-134) }\end{array}$ & 1990 & & PAILLARD, D. & 2000 \\
\hline devoir & GOSSELIN, L. (p.439-55) & 2010 & tenir & FRANÇOIS, J. & 2007 \\
\hline douter & 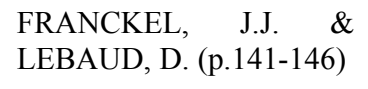 & 1990 & tirer & PAILLARD, D. & 2000 \\
\hline \multirow[t]{3}{*}{ entendre } & PICOCHE, J. & 1986 & toucher & PICOCHE, J. & 1986 \\
\hline & $\begin{array}{l}\text { FRANCKEL, } \quad \text { J.J. } \\
\text { LEBAUD, D. (p.23-56) }\end{array}$ & 1990 & & DESCLÉS, J.P. et al. & 1998 \\
\hline & $\begin{array}{l}\text { MANGUIN, J.L. \& \& } \\
\text { FRANÇOIS, J. (analyse } \\
\text { de C. Ozouf) }\end{array}$ & 2004 & & LEBAUD, D. & 2004 \\
\hline \multirow[t]{4}{*}{ jouer } & CADIOT, P. & 1999 & user & $\begin{array}{l}\text { MANGUIN, J.L. \& } \\
\text { FRANÇOIS, J. (analyse de M. } \\
\text { Sénéchal) }\end{array}$ & 2004 \\
\hline & ROMERO-LOPEZ M.C. & 2002 & venir & $\begin{array}{l}\text { FRANCKEL, J.J. \& LEBAUD, } \\
\text { D. (p.161-177) }\end{array}$ & \\
\hline & JACQUET, G. & 2003 & & HONESTE, M.L. & $\begin{array}{l}2005 \mathrm{a}, \\
2005 \mathrm{~b}\end{array}$ \\
\hline & FRANÇOIS, J. & $\begin{array}{l}2007 \mathrm{c} \text {, } \\
\text { à par. }\end{array}$ & voir & PICOCHE, J. & 1986 \\
\hline louer & PICOCHE, J. & 1986 & & $\begin{array}{l}\text { FRANCKEL, J.J. \& LEBAUD, } \\
\text { D. (p.57-70) }\end{array}$ & 1990 \\
\hline marcher & PICOCHE, J. & 1994 & & $\begin{array}{l}\text { BAT ZEEV SCHYLDKROT, } \\
\text { H. (se voir) }\end{array}$ & 1997 \\
\hline monter & DESCLÉS, J.P. et al. & 1998 & & FRANÇOIS, J. (se voir) & 2000 \\
\hline
\end{tabular}


1، (...) while crawl tends to carry with it in its various figurative manifestations the sense of limbs which by moving displace some kind of body (...), ramper focuses on the fact that the crawling entity expands and covers more and more of a surface. " (p.107)

${ }^{2}$ La propriété s'applique au sujet en construction intransitive et à l'objet en construction transitive directe.

${ }^{3}$ Je baserai ma présentation sur les articles de Desclés et al. (1998) et Desclés \& Guentcheva (2005). On trouvera dans François (2007a :14-18) une présentation détaillée de la polysémie du verbe avancer selon Desclés (2005).

${ }^{4}$ Ici c'est le cas entre les schèmes D et E, et c'est le cas également dans les analyses proposées par Desclés 2005b pour arriver et Desclés \& Guentcheva 2005 pour monter. Toutefois, dans l'analyse de la polysémie du verbe avancer, J.P. Desclés (2005a) place d'emblée à égalité les deux spécifications spatiale et temporelle.

${ }^{5}$ Cette section reprend François (2007a :28).

${ }^{6}$ Le schéma de lexis correspond dans cette théorie à la structure argumentale de J. Grimshaw ou au cadre prédicatif de S. Dik

${ }^{7}$ Le DES distingue deux entrées jouer et jouer de.

${ }^{8}$ Créer est pris dans le sens « interprète pour la première fois ». Le symbole «\# » note une disparité sémantique avec les autres synonymes de la clique.

${ }^{9}$ On ne doit pas s'attendre à ce que tous les verbes d'une clique aient obligatoirement au moins un cadre prédicatif en commun, car ce sont les verbes présentant une légère variation de construction qui permettent le 'tuilage' des cliques, c'est-à-dire qui servent de pont entre cliques.

${ }^{10}$ La visualisation comporte des sortes de « courbes de niveau ». L'élévation associée au nombre des courbes évoque le degré de compatibilité entre l'ensemble des membres de la clique et le cotexte étudié.

${ }^{11}$ La clique \{abuser, jouer, mentir, mystifier, tromper\} figure entre parenthèses parce qu'elle est associée à un type de cadre prédicatif que n'avait pas prévu l'étude antérieure de Cadiot (1999).

${ }^{12}$ Faute de place, le point de vue cartographique sur la polysémie du verbe monter ne peut pas être abordé ici. Le lecteur intéressé pourra se reporter à mon développement dans Pour une cartographie de la polysémie verbale (2007, p.204-224).

${ }^{13}$ L'attribution des rôles sémantiques est de mon fait en fonction des caractéristiques des fonctions sémantiques en cause.

${ }^{14}$ A mon sens, la notion d'intermédiaire, donc d'instrument implique le contrôle du déplacement par Jean qui sélectionne l'étage qu'il souhaite atteindre, c'est donc un agent absolument intentionnel.

${ }^{15}$ On pourrait ajouter que monter se comporte comme s'élever (la pression/la température s'élève = monte), ce qui induit une hypothèse à creuser sur la variation de sens entre se lever et s'élever.

${ }^{16} \mathrm{~J}$ 'ai indiqué sur ce tableau les prédications antonymes. Il s'avère que dans certains cas (monter un film, une affaire, un spectacle) aucune prédication antonyme n'est lexicalisée. L'action de démonter s'applique aux pièces du moteur mais pas aux scènes du film. Concernant une affaire louche, on peut sans doute dire que le juge d'instruction en démonte les rouages, mais pas qu'il la démonte, ce qui suggère qu'il est nécessaire de passer par la métaphore intermédiaire du moteur, confortant l'hypothèse dérivationnelle.

17 "the selection of a given notion as the pivot of a (universal) lexical map entails no claim at all: it is simply an arbitrary choice, the starting point before any lexical map may even begin to be drawn".

${ }^{18}$ Dictionnaire en ligne sur le site de l'ATILF-CNRS, Nancy.

${ }^{19}$ La construction causative, qui introduit le causataire comme passif, pourrait être liée à la difficulté de faire monter un chevalier en armure sur son cheval. Avec la disparition de l'armure, le cavalier devient physiquement plus autonome et la construction factitive rend mieux compte de cette autonomie.

${ }^{20}$ Le terminus a quo est fixé à la date de parution des Structures sémantiques du lexique français de Jacqueline Picoche qui a représenté un tournant dans l'étude de la polysémie en français. 